\title{
SUM RULES AND THE SZEGÖ CONDITION FOR ORTHOGONAL POLYNOMIALS ON THE REAL LINE
}

\author{
BARRY SIMON ${ }^{1}$ AND ANDREJ ZLATOŠ
}

\begin{abstract}
We study the Case sum rules, especially $C_{0}$, for general Jacobi matrices. We establish situations where the sum rule is valid. Applications include an extension of Shohat's theorem to cases with an infinite point spectrum and a proof that if $\lim n\left(a_{n}-\right.$ $1)=\alpha$ and $\lim n b_{n}=\beta$ exist and $2 \alpha<|\beta|$, then the Szegó condition fails.
\end{abstract}

\section{INTRODUCTION}

This paper discusses the relation among three objects well known to be in one-one correspondence: nontrivial (i.e., not supported on a finite set) probability measures, $\nu$, of bounded support in $\mathbb{R}$; orthogonal polynomials associated to geometrically bounded moments; and bounded Jacobi matrices. One goes from measure to polynomials via the Gram-Schmidt procedure, from polynomials to Jacobi matrices by the three-term recurrence relation, and from Jacobi matrices to measures by the spectral theorem.

We will use $J$ to denote the Jacobi matrix $\left(a_{n}>0\right)$

$$
J=\left(\begin{array}{cccc}
b_{1} & a_{1} & 0 & \ldots \\
a_{1} & b_{2} & a_{2} & \ldots \\
0 & a_{2} & b_{3} & \ldots \\
\ldots & \ldots & \ldots & \ldots
\end{array}\right)
$$

$\nu$ will normally denote the spectral measure of the vector $\delta_{1} \in \ell^{2}\left(\mathbb{Z}^{+}\right)$ and $P_{n}(x)$ the orthonormal polynomials.

We are interested in $J$ 's close to the free Jacobi matrix, $J_{0}$, with $b_{n}=0, a_{n}=1$, and $d \nu_{0}(E)=(2 \pi)^{-1} \chi_{[-2,2]} \sqrt{4-E^{2}} d E$. Most often, we will suppose $J-J_{0}$ is compact. That means $\sigma_{\text {ess }}(J)=[-2,2]$ and $J$ has only eigenvalues outside $[-2,2]$, of multiplicity one denoted $E_{j}^{ \pm}$ with $E_{1}^{+}>E_{2}^{+}>\cdots>2$ and $E_{1}^{-}<E_{2}^{-}<\cdots<-2$.

Date: May 13, 2002.

2000 Mathematics Subject Classification. Primary: 47B36; Secondary: 42C05.

${ }^{1}$ Supported in part by NSF grant DMS-9707661. 
One of the main objects of study here is the Szegö integral

$$
Z(J)=\frac{1}{2 \pi} \int_{-2}^{2} \ln \left(\frac{\sqrt{4-E^{2}}}{2 \pi d \nu_{\mathrm{ac}} / d E}\right) \frac{d E}{\sqrt{4-E^{2}}}
$$

The Szegö integral is often taken in the literature as

$$
(2 \pi)^{-1} \int_{-2}^{2} \ln \left(\frac{d \nu_{\mathrm{ac}}}{d E}\right) \frac{d E}{\sqrt{4-E^{2}}}
$$

which differs from $Z(J)$ by a constant and a critical minus sign (so the common condition that the Szegö integral not be $-\infty$ becomes $Z(J)<$ $\infty$ in our normalization). There is an enormous literature discussing when $Z(J)<\infty$ holds (see, e.g., [1, 2, 6, 8, 12, 13, 15, 16, 21, 23]). It can be shown by Jensen's inequality that $Z(J) \geq-\frac{1}{2} \ln (2)$ so the integral can only diverge to $+\infty$.

We will focus here on various sum rules that are valid. One of our main results is the following:

Theorem 1. Suppose

$$
A_{0}(J)=\lim _{N \rightarrow \infty}\left(-\sum_{n=1}^{N} \ln \left(a_{n}\right)\right)
$$

exists (although it may be $+\infty$ or $-\infty$ ). Consider the additional quantities $Z(J)$ given by (1.2) and

$$
\mathcal{E}_{0}(J)=\sum_{ \pm} \sum_{j} \ln \left[\frac{1}{2}\left(\left|E_{j}^{ \pm}\right|+\sqrt{\left(E_{j}^{ \pm}\right)^{2}-4}\right)\right]
$$

If any two of the three quantities $A_{0}(J), \mathcal{E}_{0}(J)$, and $Z(J)$ are finite, then all three are, and

$$
Z(J)=A_{0}(J)+\mathcal{E}_{0}(J)
$$

Remarks. 1. It is not hard to see that $\mathcal{E}_{0}(J)<\infty$ if and only if

$$
\sum_{ \pm} \sum_{j} \sqrt{\left(E_{j}^{ \pm}\right)^{2}-4}<\infty
$$

2. The full theorem (Theorem 4.1) does not require the limit (1.3) to exist, but is more complicated to state in that case.

3. If the three quantities are finite, many additional sum rules hold.

4. This is what Killip-Simon [10] call the $C_{0}$ sum rule.

5. Peherstorfer-Yuditskii [16] (see their remark after Lemma 2.1) prove that if $Z(J)<\infty, \mathcal{E}_{0}(J)=\infty$, then the limit in (1.3) is also infinite. 
Theorem 1 is an analog for the real line of a seventy-year old theorem for orthogonal polynomials on the unit circle:

$$
\frac{1}{2 \pi} \int_{0}^{2 \pi} \ln \left(\frac{d \nu_{a c}}{d \theta}\right) d \theta=\sum_{n=0}^{\infty} \ln \left(1-\left|\alpha_{j}\right|^{2}\right)
$$

where $\left\{\alpha_{j}\right\}_{j=1}^{\infty}$ are the Verblunsky coefficients (also called reflection, Geronimus, Schur, or Szegö coefficients) of $\nu$. This result was first proven by Verblunsky [26] in 1935, although it is closely related to Szegő's 1920 paper [23].

For $J$ 's with $J-J_{0}$ finite rank (and perhaps even with $\sum_{n=1}^{\infty} n\left(\mid a_{n}-\right.$ $\left.\left.1|+| b_{n} \mid\right)<\infty\right)$, the sum rule (1.5) is due to Case [2]. Recently, KillipSimon [10] showed how to exploit these sum rules as a spectral tool (motivated in turn by work on Schrödinger operators by Deift-Killip [4] and Denissov [5]). In particular, Killip-Simon emphasized the importance in proving sum rules on as large a class of $J$ 's as possible.

One application we will make of Theorem 1 and related ideas is to prove the following (三 Theorem 5.2):

Theorem 2. Suppose $\sigma_{\mathrm{ess}}(J) \subset[-2,2]$ and (1.6) holds. Then $Z(J)<$ $\infty$ if and only if

$$
\liminf _{N}\left(-\sum_{n=1}^{N} \ln \left(a_{n}\right)\right)<\infty
$$

Moreover, if these conditions hold, then

(i) The limit $A_{0}(J)$ in (1.3) exists and is finite.

(ii) $\lim _{N \rightarrow \infty} \sum_{n=1}^{N} b_{n}$ exists and is finite.

(iii)

$$
\sum_{n=1}^{\infty}\left(a_{n}-1\right)^{2}+\sum_{n=1}^{\infty} b_{n}^{2}<\infty
$$

Results of this genre when it is assumed that $\sigma(J)=[-2,2]$ go back to Shohat 21] with important contributions by Nevai [13]. The precise form is from Killip-Simon [10. Nikishin [15 showed how to extend this to Jacobi matrices with finitely many eigenvalues. PeherstorferYuditskii [16] proved $Z(J)<\infty$ implies (i) under the condition $\mathcal{E}_{0}(J)<$ $\infty$, allowing an infinity of eigenvalues for the first time. Our result cannot extend to situations with $\mathcal{E}_{0}(J)=\infty$ since Theorem 11 says if (i) holds and $Z(J)<\infty$, then $\mathcal{E}_{0}(J)<\infty$.

We will highlight one other result we will prove later (Corollary 6.3).

Theorem 3. Let $a_{n}, b_{n}$ be Jacobi matrix parameters so that

$$
\lim _{n \rightarrow \infty} n\left(a_{n}-1\right)=\alpha \quad \lim _{n \rightarrow \infty} n b_{n}=\beta
$$


exist and are finite. Suppose that

$$
|\beta|>2 \alpha
$$

Then $Z(J)=\infty$.

Remark. In particular, if $\alpha<0$, (1.11) always holds. (1.11) describes three-quarters of the $(2 \alpha, \beta)$ plane.

In Section 6, we will discuss the background for this result, and describe results of Zlatoš [27 that show if $|\beta| \leq 2 \alpha$ and one has additional information on the approach to the limit (1.10), then $Z(J)<\infty$. Thus Theorem 3 captures the precise region where one has (1.10) and one can hope to prove $Z(J)=\infty$.

Theorem 3 will actually follow from a more general result (see Theorem 4.4, 6.1, and 6.2).

Theorem 4. Suppose (1.9) holds and that either $\lim \sup \left(-\sum_{j=1}^{n}\left(a_{j}-\right.\right.$ $\left.\left.1+\frac{1}{2} b_{j}\right)\right)=\infty$ or $\lim \sup \left(-\sum_{j=1}^{n}\left(a_{j}-1-\frac{1}{2} b_{j}\right)\right)=\infty$. Then $Z(J)=\infty$.

The main technique in this paper exploits the $m$-function, the Borel transform of the measure, $\nu$ :

$$
m_{\nu}(E)=\int \frac{d \nu(x)}{x-E}
$$

Since $\nu$ is supported on $[-2,2]$ plus the set of points $\left\{E_{j}^{ \pm}\right\}$, we can write

$$
m_{\nu}(E)=\sum_{ \pm} \sum_{j} \frac{\nu\left(\left\{E_{j}^{ \pm}\right\}\right)}{E_{j}^{ \pm}-E}+\int_{-2}^{2} \frac{d \nu(x)}{x-E}
$$

It is useful to transfer everything to the unit circle, using the fact that $z \mapsto E=z+z^{-1}$ maps $\mathbb{D}=\{z|| z \mid<1\}$ onto the cut plane $\mathbb{C} \backslash[-2,2]$. Thus we can define for $|z|<1$

$$
M(z)=-m_{\nu}\left(z+z^{-1}\right)
$$

The minus sign is picked so $\operatorname{Im} M(z)>0$ if $\operatorname{Im} z>0$. We use $M(z ; J)$ when we want to make the $J$-dependence explicit.

In (1.13), we translate the pole term directly. Define $\beta_{j}$ so

$$
E_{j}^{ \pm}=\beta_{j}^{ \pm}+\left(\beta_{j}^{ \pm}\right)^{-1}
$$

with $\left|\beta_{j}^{ \pm}\right|>1$. We sometimes drop the explicit \pm symbol and count the $\beta_{j}$ 's in one set. We define a signed measure $d \mu^{\#}$ on $(0,2 \pi)$ by $\operatorname{Im} M\left(r e^{i \theta}\right) \rightarrow d \mu^{\#}$ weakly as $r \uparrow 1 . \mu^{\#}$ is positive on $(0, \pi)$ and 
negative on $(\pi, 2 \pi)$. Thus (1.13) implies

$$
\operatorname{Im} M(z)=\operatorname{Im} \sum_{j} \frac{\mu\left(\left\{\beta_{j}^{-1}\right\}\right)}{z+z^{-1}-\left(\beta_{j}+\beta_{j}^{-1}\right)}+\frac{1}{2 \pi} \int_{0}^{2 \pi} P\left(z, e^{i \varphi}\right) d \mu^{\#}(\varphi)
$$

where we use $\mu\left(\left\{\beta_{j}^{-1}\right\}\right)$ for the weights $\nu\left(\left\{E_{j}\right\}\right)$ and $P(z, w)$, with $|z| \leq$ $1,|w|=1$, is the Poisson kernel

or

$$
P(z, w)=\frac{1-|z|^{2}}{|z-w|^{2}}
$$

$$
P\left(r e^{i \theta}, e^{i \varphi}\right) \equiv P_{r}(\theta-\varphi)=\frac{1-r^{2}}{1+r^{2}-2 r \cos (\theta-\varphi)}
$$

We note now that since $\mu\left(\left\{\beta_{j}^{-1}\right\}\right)$ are point mass of a probability measure, we have

$$
\sum_{j} \mu\left(\left\{\beta_{j}^{-1}\right\}\right) \leq 1
$$

It is useful to use the fact that $\mu^{\#}$ is odd under reflection to rewrite (1.16) in the form

$\operatorname{Im} M\left(r e^{i \theta}\right)=\operatorname{Im} \sum_{j} \frac{\mu\left(\left\{\beta_{j}^{-1}\right\}\right)}{r e^{i \theta}+r^{-1} e^{-i \theta}-\left(\beta_{j}+\beta_{j}^{-1}\right)}+\frac{1}{2 \pi} \int_{0}^{\pi} D_{r}(\theta, \varphi) d \mu(\varphi)$

where

$$
D_{r}(\theta, \varphi)=P_{r}(\theta, \varphi)-P_{r}(\theta,-\varphi)
$$

This is because $M(z)=\overline{M(\bar{z})}$, so that $\mu^{\#} \uparrow[-\pi, 0]$ is a reflection across $\mathbb{R}$ of $\mu \equiv \mu^{\#} \uparrow[0, \pi]$.

Note that not only does $\operatorname{Im} M\left(r e^{i \theta}\right) d \theta$ converge weakly to $\mu^{\#}$, but by general principles [20], $\lim _{r \uparrow 1} M\left(r e^{i \theta}\right) \equiv M\left(e^{i \theta}\right)$ exists for a.e. $\theta$ and $d \mu_{\mathrm{ac}}^{\#}=\operatorname{Im} M\left(e^{i \theta}\right) d \theta$.

Section 2, the technical core of the paper, proves some convergence results about integrals of $\ln \left[\operatorname{Im} M\left(r e^{i \theta}\right)\right]$. It is precisely such integrals that arise in Section 3 where, following Killip-Simon [10], we use the well-known

$$
-m(z ; J)^{-1}=z-b_{1}+a_{1}^{2} m\left(z ; J^{(1)}\right)
$$

where $J^{(1)}$ is $J$ with the top row and leftmost column removed. We will be able to prove sum rules that compare $J$ and $J^{(1)}$. In Section 4 , we will then list various sum rules, including Theorems 1 and 4. Section 5 
proves Theorem 2 and Section 6 discusses Coulomb Jacobi matrices $\left(J-J_{0}\right.$ decays as $\left.n^{-1}\right)$ and Theorem 3 in particular.

It is a pleasure to thank Mourad Ismail, Rowan Killip, and Paul Nevai for useful discussions.

\section{Continuity of Integrals of $\ln (\operatorname{Im} M)$}

In this section, we will prove a general continuity result about boundary values of interest for $M$-functions of the type defined in (1.18). We will consider suitable weight functions, $w(\varphi)$, on $[0, \pi]$, of which the examples of most interest are $w(\varphi)=\sin ^{k}(\varphi), k=0$ or 2. Our goal is to prove that

$$
\lim _{r \uparrow 1} \int \ln \left[\operatorname{Im} M\left(r e^{i \varphi}\right)\right] w(\varphi) d \varphi=\int \ln \left[\operatorname{Im} M\left(e^{i \varphi}\right)\right] w(\varphi) d \varphi
$$

and that the convergence is in $L^{1}$ if the integral on the right is finite. All integrals in this section are from 0 to $\pi$ if not indicated otherwise. We define

$$
d(\varphi) \equiv \min (\varphi, \pi-\varphi)
$$

and we suppose that

$$
0 \leq w(\varphi) \leq C_{1} d(\varphi)^{-1+\alpha}
$$

for some $C_{1}, \alpha>0$ and that $w$ is $C^{1}$ with

$$
\left|w^{\prime}(\varphi) w(\varphi)^{-1}\right| \leq C_{2} d(\varphi)^{-\beta}
$$

for $C_{2}, \beta>0$. For weights of interest, one can take $\alpha=\beta=1$.

Remarks. 1. For the applications in mind, we are only interested in allowing "singularities" (i.e., $w$ vanishing or going to infinity) at 0 or $\pi$, but all results hold with unchanged proofs if $d(\varphi) \equiv \min \left\{\left|\varphi-\varphi_{j}\right|\right\}$ for any finite set $\left\{\varphi_{j}\right\}$. For example, $w(\varphi)=\sin ^{2}(m \varphi)$ as in 11 is fine.

2. Note that by (2.3), $\int_{0}^{\pi} w(\varphi) d \varphi<\infty$.

The main technical result we will need is:

Theorem 2.1. Let $M$ be a function with a representation of the form (1.19) and let $w$ be a weight function obeying (2.3) and (2.4). Then (2.1) holds. Moreover, if

$$
\int \ln \left[\operatorname{Im} M\left(e^{i \varphi}\right)\right] w(\varphi) d \varphi>-\infty
$$

(it is never $+\infty$ ), then

$$
\lim _{r \uparrow 1} \int\left|\ln \left[\operatorname{Im} M\left(r e^{i \varphi}\right)\right]-\ln \left[\operatorname{Im} M\left(e^{i \varphi}\right)\right]\right| w(\varphi) d \varphi=0
$$


Let $\ln _{ \pm}$be defined by

$$
\ln _{ \pm}(y)=\max (0, \pm \ln (y))
$$

SO

$$
\begin{aligned}
\ln (y) & =\ln _{+}(y)-\ln _{-}(y) \\
|\ln (y)| & =\ln _{+}(y)+\ln _{-}(y)
\end{aligned}
$$

We will prove Theorem 2.1 by proving

Theorem 2.2. For any $a>0$ and $p<\infty, \ln _{+}\left[\operatorname{Im}\left(M\left(e^{i \varphi}\right)\right) / a\right] \in$ $L^{p}((0, \pi), w(\varphi) d \varphi)$, and

$$
\lim _{r \uparrow 1} \int\left|\ln _{+}\left(\frac{\operatorname{Im} M\left(r e^{i \varphi}\right)}{a}\right)-\ln _{+}\left(\frac{\operatorname{Im} M\left(e^{i \varphi}\right)}{a}\right)\right|^{p} w(\varphi) d \varphi=0
$$

Theorem 2.3. For any $a>0$, we have

$$
\lim _{r \uparrow 1} \int \ln _{-}\left(\frac{\operatorname{Im} M\left(r e^{i \varphi}\right)}{a}\right) w(\varphi) d \varphi=\int \ln _{-}\left(\frac{\operatorname{Im} M\left(e^{i \varphi}\right)}{a}\right) w(\varphi) d \varphi
$$

Proof of Theorem 2.1 given Theorems 2.9 and 2.3. By Fatou's lemma and the fact that for a.e. $\varphi, \operatorname{Im} M\left(r e^{i \varphi}\right) \rightarrow \operatorname{Im} M\left(e^{i \varphi}\right)$, we have

$$
\underset{r \uparrow 1}{\liminf } \int \ln _{-}\left[\operatorname{Im} M\left(r e^{i \varphi}\right)\right] w(\varphi) d \varphi \geq \int \ln _{-}\left[\operatorname{Im} M\left(e^{i \varphi}\right)\right] w(\varphi) d \varphi
$$

Since Theorem 2.2 says that $\sup _{0<r \leq 1} \int \ln _{+}\left[\operatorname{Im} M\left(r e^{i \varphi}\right)\right] w(\varphi) d \varphi<\infty$, it follows that if $\int \ln _{-}\left[\operatorname{Im} M\left(e^{i \varphi}\right)\right] w(\varphi) d \varphi=\infty$, then (2.1) holds.

If (2.5) holds, then

$$
\lim _{a \downarrow 0} \int \ln _{-}\left[\frac{\operatorname{Im} M\left(e^{i \varphi}\right)}{a}\right] w(\varphi) d \varphi=0
$$

since $\ln _{-}(y / a)$ is monotone decreasing to 0 as $a$ decreases. Given $\varepsilon$, first find $a$ so

$$
\int \ln _{-}\left[\frac{\operatorname{Im} M\left(e^{i \varphi}\right)}{a}\right] w(\varphi) d \varphi<\frac{\varepsilon}{3}
$$

and then, by (2.8), $r_{1}<1$ so for $r_{1}<r<1$,

$$
\int \ln _{-}\left[\frac{\operatorname{Im} M\left(r e^{i \varphi}\right)}{a}\right] w(\varphi) d \varphi<\frac{\varepsilon}{3}
$$

By (2.7), find $r_{2}<1$, so for $r_{2}<r<1$,

$$
\int\left|\ln _{+}\left[\frac{\operatorname{Im} M\left(r e^{i \varphi}\right)}{a}\right]-\ln _{+}\left[\frac{\operatorname{Im} M\left(e^{i \varphi}\right)}{a}\right]\right| w(\varphi) d \varphi<\frac{\varepsilon}{3}
$$


Writing

$$
|\ln (\alpha)-\ln (\beta)| \leq\left|\ln _{+}\left(\frac{\alpha}{a}\right)-\ln _{+}\left(\frac{\beta}{a}\right)\right|+\ln _{-}\left(\frac{\alpha}{a}\right)+\ln _{-}\left(\frac{\beta}{a}\right)
$$

we see that if $\max \left(r_{1}, r_{2}\right)<r<1$, then

$$
\int\left|\ln \left[\operatorname{Im} M\left(r e^{i \varphi}\right)\right]-\ln \left[\operatorname{Im} M\left(e^{i \varphi}\right)\right]\right| w(\varphi) d \varphi<\varepsilon
$$

so (2.6) holds.

We will prove Theorem 2.2 by using the dominated convergence theorem and standard maximal function techniques. Given the measure $\mu$ on $(0, \pi)$, we define $\tilde{\mu}$ to be the sum of $\mu$ and its reflection on $(\pi, 2 \pi)$, and its maximal function by

$$
\mu^{*}(x)=\sup _{0<a<\pi} \frac{\tilde{\mu}(x-a, x+a)}{2 a}
$$

The Hardy-Littlewood maximal inequality for measures (see Rudin [20]) says that

$$
\left|\left\{x \mid \mu^{*}(x)>\lambda\right\}\right| \leq \frac{3 \tilde{\mu}(0, \pi)}{\lambda}
$$

Lemma 2.4. Let $M$ be an $M$-function based on a measure $\mu$ on $[0, \pi]$ and weights at the poles at $\left(\beta_{j}^{ \pm}\right)^{-1}$, and let $\alpha$ be a sum of the weights of the poles. Then for $0<r<1$,

$$
\operatorname{Im} M\left(r e^{i \theta}\right) \leq \mu^{*}(\theta)+\alpha r^{-1}[\sin (\theta)]^{-2}
$$

Proof. Since $D_{r}(\theta, \varphi) \leq P_{r}(\theta, \varphi)$ and $P_{r}$ is a convolution operator with a positive even decreasing function of $\varphi$ on $[0, \pi]$ with $\int_{0}^{2 \pi} P_{r}(\varphi) d \varphi / 2 \pi=$ 1 , we have, by standard calculations, that

$$
\int_{0}^{\pi} D_{r}(\theta, \varphi) \frac{d \mu(\varphi)}{2 \pi} \leq \mu^{*}(\theta)
$$

On the other hand, for $\beta \geq 1$,

$$
\left|\frac{1}{z+z^{-1}-\beta-\beta^{-1}}\right|=\left|\frac{z}{(z-\beta)\left(z-\beta^{-1}\right)}\right| \leq \frac{|z|}{|\operatorname{Im} z|^{2}} \leq \frac{1}{r \sin ^{2} \theta}
$$

if $z=r e^{i \theta}$, so summing the pole term shows,

$$
\operatorname{Im} \sum_{i} \frac{\mu\left(\left\{\beta_{i}^{-1}\right\}\right)}{z+z^{-1}-\beta_{i}-\beta_{i}^{-1}} \leq \frac{\sum_{i} \mu\left(\left\{\beta_{i}^{-1}\right\}\right)}{r \sin ^{2} \theta}
$$


Proof of Theorem 2.2. Let

$$
f_{1}(\theta)=\mu^{*}(\theta) \quad f_{2}(\theta)=2[\sin (\theta)]^{-2}
$$

For a.e. $\theta, \ln _{+}\left[\left(\operatorname{Im} M\left(r e^{i \theta}\right)\right) / a\right] \rightarrow \ln _{+}\left[\left(\operatorname{Im} M\left(e^{i \theta}\right)\right) / a\right]$. By (2.11) for all $\frac{1}{2}<r<1, \ln _{+}\left[\left(\operatorname{Im} M\left(r e^{i \theta}\right)\right) / a\right] \leq \ln _{+}\left[\left(f_{1}(\theta)+f_{2}(\theta)\right) / a\right]$. Thus if we prove that for all $p<\infty$,

$$
\int\left|\ln _{+}\left(\frac{f_{1}+f_{2}}{a}\right)\right|^{p} w(\varphi) d \varphi<\infty
$$

we obtain (2.7) by the dominated convergence theorem. Since

$$
\left|\ln _{+}(x)\right|^{p} \leq C(p, q)|x|^{q}
$$

for any $p<\infty, q>0$, and suitable $C(p, q)$ and

$$
|x+y|^{q} \leq 2^{q}|x|^{q}+2^{q}|y|^{q}
$$

it suffices to find some $q>0$, so

$$
\int\left(\left|f_{1}(\varphi)\right|^{q}+\left|f_{2}(\varphi)\right|^{q}\right) w(\varphi) d \varphi<\infty
$$

Since for $v^{-1}+t^{-1}=1$,

$$
\int\left|f_{1}(\varphi)\right|^{q} w(\varphi) d \varphi \leq\left(\int\left|f_{1}(\varphi)\right|^{q v} d \varphi\right)^{1 / v}\left(\int|w(\varphi)|^{t} d \varphi\right)^{1 / t}
$$

and $w(\varphi) \in L^{t}$ for some $t>1$ by (2.3), it suffices to find some $s>0$ with

$$
\int\left(\left|f_{1}(\varphi)\right|^{s}+\left|f_{2}(\varphi)\right|^{s}\right) d \varphi<\infty
$$

By (2.10), $\int\left|f_{1}(\varphi)\right|^{s} d \varphi<\infty$ if $s<1$ and clearly, $\int\left|f_{2}(\varphi)\right|^{s} d \varphi<\infty$ if $s<\frac{1}{2}$.

As a preliminary to the proof of Theorem 2.3, we need

Lemma 2.5. Let $w$ obey (2.4). Let $0<\varphi_{0}<\pi$ and let $\varphi_{1}, \varphi_{2} \in[0, \pi]$ obey

(a) $\quad d\left(\varphi_{1}\right) \geq d\left(\varphi_{0}\right), \quad d\left(\varphi_{2}\right) \geq d\left(\varphi_{0}\right)$

(b) $\quad\left|\varphi_{1}-\varphi_{2}\right| \leq d\left(\varphi_{0}\right)^{\beta}$

Then for $C_{3}=C_{2} \exp \left(C_{2}\right)$,

$$
\left|\frac{w\left(\varphi_{1}\right)}{w\left(\varphi_{2}\right)}-1\right| \leq C_{3}\left|\varphi_{1}-\varphi_{2}\right| d\left(\varphi_{0}\right)^{-\beta}
$$


Proof.

$$
\begin{aligned}
\left|\frac{w\left(\varphi_{1}\right)}{w\left(\varphi_{2}\right)}-1\right| & =\left|\exp \left(\int_{\varphi_{1}}^{\varphi_{2}} \frac{w^{\prime}(\eta)}{w(\eta)} d \eta\right)-1\right| \\
& \leq\left|\exp \left(C_{2}\left|\varphi_{2}-\varphi_{1}\right| d\left(\varphi_{0}\right)^{-\beta}\right)-1\right|
\end{aligned}
$$

by (2.4) and (2.13). But $\left|e^{x}-1\right| \leq e^{|x|}|x|$, so by (2.14),

$$
\left|\frac{w\left(\varphi_{1}\right)}{w\left(\varphi_{2}\right)}-1\right| \leq C_{2} \exp \left(C_{2}\right)\left|\varphi_{1}-\varphi_{2}\right| d\left(\varphi_{0}\right)^{-\beta}
$$

which is (2.15).

We will also need the following pair of lemmas:

Lemma 2.6. Let $0<\eta<\theta<\pi-\eta$ and

$$
N_{r}(\theta, \eta)=\int_{\theta-\eta}^{\theta+\eta} D_{r}(\theta, \varphi) \frac{d \varphi}{2 \pi}
$$

Then

$$
0 \leq\left[1-N_{r}(\theta, \eta)\right] \leq \frac{2(1-r)}{r \sin ^{2} \theta}+\frac{1-r}{r \sin ^{2} \eta}
$$

Proof. We have

$$
1=\int_{0}^{2 \pi} P_{r}(\theta, \varphi) \frac{d \varphi}{2 \pi}
$$

so since $D_{r} \leq P_{r}, N_{r} \leq 1$ and

$$
1-N_{r}(\theta, \eta) \leq \frac{2}{2 \pi} \int_{-\pi}^{0} P_{r}(\theta, \varphi) d \varphi+\frac{1}{2 \pi} \int_{\substack{\varphi \in[0, \pi] \\|\theta-\varphi| \geq \eta}} P_{r}(\theta, \varphi) d \varphi
$$

Now

$$
\begin{aligned}
P_{r}(\theta, \varphi) & =\frac{1-r^{2}}{(1-r)^{2}+4 r \sin ^{2}\left[\frac{1}{2}(\theta-\varphi)\right]} \\
& \leq \frac{2(1-r)}{4 r \sin ^{2}\left[\frac{1}{2}(\theta-\varphi)\right]} \\
& \leq \frac{2(1-r)}{r \sin ^{2}(\theta-\varphi)}
\end{aligned}
$$

The first integrand in $(2.18)$ is thus bounded by $2 r^{-1}(1-r)\left[\sin ^{2}(\theta)\right]^{-1}$ and the second by $2 r^{-1}(1-r)\left[\sin ^{2}(\eta)\right]^{-1}$, so $(2.17)$ is immediate.

Lemma 2.7. If $\int_{0}^{\pi} \operatorname{Im} M\left(e^{i \theta}\right) d \theta \neq 0$, then for $\theta \in[0, \pi], r \in\left(\frac{1}{2}, 1\right)$,

$$
\operatorname{Im} M\left(r e^{i \theta}\right) \geq c\left(r^{-1}-r\right) \sin \theta
$$


Proof. In terms of the real line $m$ function, for $E_{2}>0, E_{1}$ real,

$$
\operatorname{Im}\left[-m\left(E_{1}-i E_{2}\right)\right] \geq \frac{E_{2}}{\pi} \int_{-2}^{2} \frac{\operatorname{Im} m(E) d E}{\left(E_{1}-E\right)^{2}+E_{2}^{2}}
$$

since we have dropped the positive contributions of $\nu_{\text {sing }}$ to $\operatorname{Im}(-m)$. Now if $z=r e^{i \theta}$,

$$
M(z)=-m\left(E_{1}-i E_{2}\right)
$$

where $z+z^{-1}=E_{1}-i E_{2}$ or $E_{1}=\left(r+r^{-1}\right) \cos \theta, E_{2}=\left(r^{-1}-r\right) \sin \theta$. If $r>\frac{1}{2}$, then $\left|E_{1}\right| \leq \frac{5}{2},\left|E_{2}\right| \leq \frac{3}{2}$, and in (2.21), $|E| \leq 2$. Thus

$$
\operatorname{Im} M(z) \geq c E_{2}(z)
$$

which is 2.20$)$.

Proof of Theorem 2.3. Since $\mathrm{ln}_{-}$is a decreasing function, to get upper bounds on $\ln _{-}\left[\operatorname{Im} M\left(r e^{i \theta}\right) / a\right]$, we can use a lower bound on $\operatorname{Im} M$. The elementary bound

$$
\ln _{-}(a b) \leq \ln _{-}(a)+\ln _{-}(b)
$$

will be useful.

As already noted, Fatou's lemma implies the lim inf of the left side of (2.8) is bounded from below by the right side, so it suffices to prove that

$\limsup _{r \uparrow 1} \int_{0}^{\pi} \ln _{-}\left(\frac{\operatorname{Im} M\left(r e^{i \varphi}\right)}{a}\right) w(\varphi) d \varphi \leq \int_{0}^{\pi} \ln _{-}\left(\frac{\operatorname{Im} M\left(e^{i \varphi}\right)}{a}\right) w(\varphi) d \varphi$

Pick $\gamma$ and $\kappa$ so $0<\max (\beta, 1) \gamma<\kappa<\frac{1}{2}$ and let $\theta_{0}(r)=(1-r)^{\gamma}$, $\eta(r)=(1-r)^{\kappa}$. We will bound $\operatorname{Im} M\left(r e^{i \theta}\right)$ from below for $d(\theta) \leq \theta_{0}(r)$ using (2.20), and for $d(\theta) \geq \theta_{0}(r)$, we will use the Poisson integral for the region $|\varphi-\theta| \leq \eta(r)$.

By $(2.20$ and $(2.3)$,

$$
\int_{d(\varphi) \leq \theta_{0}(r)} \ln _{-}\left(\frac{\operatorname{Im} M\left(r e^{i \varphi}\right)}{a}\right) w(\varphi) d \varphi \leq C_{a} \theta_{0}^{\alpha}\left[\ln _{-}\left(r^{-1}-r\right)+\ln _{-} \theta_{0}\right]
$$

which goes to zero as $r \uparrow 1$ for any $a$. So suppose $d(\theta)>\theta_{0}$. Write

$$
\begin{aligned}
\operatorname{Im} M\left(r e^{i \theta}\right) & \geq \int_{\theta-\eta(r)}^{\theta+\eta(r)} D_{r}(\theta, \varphi) \operatorname{Im} M\left(e^{i \varphi}\right) \frac{d \varphi}{2 \pi} \\
& =N_{r}(\theta, \eta) \int_{\theta-\eta(r)}^{\theta+\eta(r)} \frac{D_{r}(\theta, \varphi)}{2 \pi N_{r}(\theta, \eta)} \operatorname{Im} M\left(e^{i \varphi}\right) d \varphi
\end{aligned}
$$

For later purposes, note that for $d(\theta)>\theta_{0},(2.17)$ implies

$$
0 \leq 1-N_{r}(\theta, \eta) \leq C(1-r)^{1-2 \kappa}
$$


which goes to zero since $\kappa<\frac{1}{2}$. Using (2.24) and (2.22), we bound $\ln _{-}\left[\operatorname{Im} M\left(r e^{i \theta}\right) / a\right]$ as two $\ln _{-}$'s. Since $\ln _{-}$is convex and $D_{r}(\theta, \varphi) / 2 \pi N_{r}(\theta, \eta)$ $\chi_{(\theta-\eta, \theta+\eta)}(\varphi) d \varphi$ is a probability measure, we can use Jensen's inequality to see that

$$
\begin{aligned}
w(\theta) & \ln _{-}\left[\frac{\operatorname{Im} M\left(r e^{i \theta}\right)}{a}\right] \\
& \leq w(\theta) \ln _{-}\left[N_{r}(\theta, \eta)\right]+\int_{\theta-\eta(r)}^{\theta+\eta(r)} \frac{w(\theta)}{w(\varphi)} \frac{D_{r}(\theta, \varphi)}{N_{r}(\theta, \eta)} w(\varphi) \ln _{-}\left[\frac{\operatorname{Im} M\left(e^{i \varphi}\right)}{a}\right] \frac{d \varphi}{2 \pi}
\end{aligned}
$$

In the first term for the $\theta$ 's with $d(\theta) \geq \theta_{0}(r), N_{r}$ obeys (2.25) so

$$
\int_{d(\theta) \geq \theta_{0}(r)} w(\theta) \ln _{-}\left[N_{r}(\theta, \eta)\right] d \theta=O\left((1-r)^{1-2 \kappa}\right) \rightarrow 0
$$

In the second term, note that for the $\theta$ 's in question, $N_{r}(\theta, \eta)^{-1}-1=$ $O\left((1-r)^{1-2 \kappa}\right)$ and by (2.15), $w(\theta) / w(\varphi)-1=O\left((1-r)^{\kappa-\beta \gamma}\right)$. Since $D_{r}(\theta, \varphi) \leq P_{r}(\theta, \varphi)$, we thus have

$$
\begin{aligned}
& \int_{d(\theta) \geq \theta_{0}} \ln _{-}\left[\frac{\operatorname{Im} M\left(r e^{i \theta}\right)}{a}\right] w(\theta) d \theta \\
& \leq O\left((1-r)^{1-2 \kappa}\right)+\left[1+O\left((1-r)^{1-2 \kappa}\right)\right]\left[1+O\left((1-r)^{\kappa-\beta \gamma}\right)\right] \\
& \quad \int_{\substack{d(\theta) \geq \theta_{0} \\
|\varphi-\theta| \leq \eta}} P_{r}(\theta, \varphi) w(\varphi) \ln \ln _{-}\left[\frac{\operatorname{Im} M\left(e^{i \varphi}\right)}{a}\right] d \varphi \frac{d \theta}{2 \pi}
\end{aligned}
$$

Since the integrand is positive, we can extend it to $\{(\theta, \varphi) \mid \theta \in$ $[0,2 \pi], \varphi \in[0, \pi]\}$ and do the $\theta$ integration using $\int P_{r}(\theta, \varphi) d \theta / 2 \pi=1$. The result is (2.23).

This concludes the proof of Theorem 2.1. By going through the proof, one easily sees that

Theorem 2.8. Theorem 2.1 remains true if in (2.1) and (2.6), $\ln \left[\operatorname{Im} M\left(r e^{i \varphi}\right)\right]$ is replaced by $\ln \left[g(r) \sin \varphi+\operatorname{Im} M\left(r e^{i \varphi}\right)\right]$ where $g(r) \geq 0$ and $g(r) \rightarrow 0$ as $r \uparrow 1$.

Proof. In the $\ln _{+}$bounds, we get an extra $\left[\sup _{\frac{1}{2}<r<1} g(r)\right] \sin \theta$ in $f_{2}(\theta)$. Since we still have pointwise convergence, we easily get the analog of Theorem 2.2. In the proof of Theorem 2.3, Fatou is unchanged since $g(r) \rightarrow 0$, and since

$$
\ln _{-}\left(g(r) \sin \varphi+\operatorname{Im} M\left(r e^{i \varphi}\right)\right) \leq \ln _{-}\left(\operatorname{Im} M\left(r e^{i \varphi}\right)\right)
$$

the limsup bound has an unchanged proof. 


\section{The Step-By-Step Sum Rules}

We will call $J$ a BW matrix (for Blumenthal-Weyl) if $J$ is a bounded Jacobi matrix with $\sigma_{\text {ess }}(J)=[-2,2]$, for example, if $J-J_{0}$ is compact. Let $J^{(n)}$ be the matrix resulting from removing the first $n$ rows and columns. Let $\left\{E_{j}^{ \pm}(J)\right\}_{j=1}^{\infty}$ be the eigenvalues of $J$ above/below \pm 2 , ordered by $\pm E_{1}^{ \pm} \geq \pm E_{2}^{ \pm} \geq \cdots$ with $E_{j}^{ \pm}(J)$ defined to be \pm 2 if there are only finitely many eigenvalues $k<j$ above/below \pm 2 . Then by the min-max principle,

$$
\pm E_{j+n}^{ \pm}(J) \leq \pm E_{j}^{ \pm}\left(J^{(n)}\right) \leq \pm E_{j}^{ \pm}(J)
$$

We have $\lim _{j \rightarrow \infty} E_{j}^{ \pm}(J)= \pm 2$ if $J$ is a BW matrix.

It follows by the convergence of sums of alternating series that if $f$ is even or odd and monotone on $[2, \infty)$ with $f(2)=0$, then

$$
\lim _{N \rightarrow \infty} \sum_{ \pm} \sum_{j=1}^{N}\left[f\left(E_{j}^{ \pm}(J)\right)-f\left(E_{j}^{ \pm}\left(J^{(n)}\right)\right)\right] \equiv \delta f_{n}(J)
$$

exists and is finite. If $\beta_{j}^{ \pm}$is defined by $E_{j}^{ \pm}=\beta_{j}^{ \pm}+\left(\beta_{j}^{ \pm}\right)^{-1}$ with $\left|\beta_{j}\right|>1$, we define $X_{\ell}^{(n)}(J)$ as $\delta f_{n}(J)$ for

$$
f(E)= \begin{cases}\ln |\beta| & \ell=0 \\ -\frac{1}{\ell}\left[\beta^{\ell}-\beta^{-\ell}\right] & \ell=1,2 \ldots\end{cases}
$$

In addition, we will need

$$
\zeta_{\ell}^{(n)}(J)= \begin{cases}-\sum_{j=1}^{n} \ln \left(a_{j}\right) & \ell=0 \\ \frac{2}{\ell} \lim _{m \rightarrow \infty}\left[\operatorname{Tr}\left(T_{\ell}\left(\frac{1}{2} J_{m ; F}\right)\right)-\operatorname{Tr}\left(T_{\ell}\left(\frac{1}{2} J_{m-n ; F}^{(n)}\right)\right]\right. & \ell=1,2, \ldots\end{cases}
$$

where $J_{m ; F}$ is the finite matrix formed from the first $m$ rows and columns of $J$ and $T_{\ell}$ is the $\ell$-th Chebyshev plynomial (of the first kind). As noted in [10, Proposition 4.3], the limit in (3.4) exists since the expression is independent of $m$ once $m>\ell+n$.

Note that

$$
\begin{aligned}
\zeta_{1}^{(n)}(J) & =\sum_{j=1}^{n} b_{j} \\
\zeta_{2}^{(n)}(J) & =\sum_{j=1}^{n} \frac{1}{2} b_{j}^{2}+\left(a_{j}^{2}-1\right)
\end{aligned}
$$

as computed in [10]. 
Note that by construction (with $J^{(0)} \equiv J$ ),

$$
X_{\ell}^{(n)}(J)=\sum_{j=0}^{n-1} X_{\ell}^{(1)}\left(J^{(j)}\right)
$$

and

$$
\zeta_{\ell}^{(n)}(J)=\sum_{j=0}^{n-1} \zeta_{\ell}^{(1)}\left(J^{(j)}\right)
$$

As final objects we need

$$
Z(J)=\frac{1}{4 \pi} \int_{0}^{2 \pi} \ln \left(\frac{\sin \theta}{\operatorname{Im} M\left(e^{i \theta}, J\right)}\right) d \theta
$$

and for $\ell \geq 1$,

$$
\begin{aligned}
Z_{\ell}^{ \pm}(J) & =\frac{1}{4 \pi} \int_{0}^{2 \pi} \ln \left(\frac{\sin \theta}{\operatorname{Im} M\left(e^{i \theta}, J\right)}\right)(1 \pm \cos (\ell \theta)) d \theta \\
Y_{\ell}(J) & =-\frac{1}{2 \pi} \int_{0}^{2 \pi} \ln \left(\frac{\sin \theta}{\operatorname{Im} M\left(e^{i \theta}, J\right)}\right) \cos (\ell \theta) d \theta
\end{aligned}
$$

We include " $\sin \theta$ " inside $\ln (\ldots)$ so that $Z\left(J_{0}\right)=Z_{\ell}^{ \pm}\left(J_{0}\right)=Y_{\ell}\left(J_{0}\right)=0$ because $M\left(z, J_{0}\right)=z$.

Of course,

$$
Z_{\ell}^{ \pm}(J)=Z(J) \mp \frac{1}{2} Y_{\ell}(J)
$$

when all integrals converge. By Theorem 2.2, the $\ln _{-}$piece of the integrals in (3.9)-(3.11) always converges. Since $1 \pm \cos (\ell \theta) \geq 0$, the integrals defining $Z(J), Z_{\ell}^{ \pm}(J)$ either converge or diverge to $+\infty$. We therefore always define $Z(J)$ and $Z_{\ell}^{ \pm}(J)$ although they may take the value $+\infty$. Since $[1 \pm \cos (\ell \theta)] \leq 2, Z(J)<\infty$ implies $Z_{\ell}^{ \pm}(J)<\infty$, so we define $Y_{\ell}(J)$ by (3.12) if and only if $Z(J)<\infty$.

If $Z(J)<\infty$, we say $J$ obeys the Szegő condition or $J$ is Szegő. If $Z_{1}^{ \pm}(J)<\infty$, we say $J$ is Szegő at \pm 2 since, for example, if $Z_{1}^{+}(J)<\infty$, the integral in (3.9) converges near $\theta=0(E=2 \cos (\theta)$ near +2$)$ and if $Z_{1}^{-}(J)<\infty$, the integral converges near $\theta=\pi$ (i.e., $E=-2$ ). Note that while $Z_{1}^{+}(J)<\infty$ only implies convergence of (3.9) at $\theta=0$, it also implies that at $\theta=\pi$ the integral with a $\sin ^{2} \theta$ inserted converges (quasi-Szegő condition).

Our main goal in this section is to prove the next three theorems

Theorem 3.1 (Step-by-Step Sum Rules). Let $J$ be a $B W$ matrix. $Z(J)<$ $\infty$ if and only if $Z\left(J^{(1)}\right)<\infty$, and if $Z(J)<\infty$, we have

$$
Z(J)=-\ln \left(a_{1}\right)+X_{0}^{(1)}(J)+Z\left(J^{(1)}\right)
$$




$$
Y_{\ell}(J)=\zeta_{\ell}^{(1)}(J)+X_{\ell}^{(1)}(J)+Y_{\ell}\left(J^{(1)}\right) ; \quad \ell=1,2,3, \ldots
$$

Remarks. 1. By iteration and (3.7)/(3.8), we obtain if $Z(J)<\infty$, then $Z\left(J^{(n)}\right)<\infty$ and

$$
\begin{aligned}
Z(J) & =-\sum_{j=1}^{n} \ln \left(a_{j}\right)+X_{0}^{(n)}(J)+Z\left(J^{(n)}\right) \\
Y_{\ell}(J) & =\zeta_{\ell}^{(n)}(J)+X_{\ell}^{(n)}(J)+Y_{\ell}\left(J^{(n)}\right) ; \quad \ell=1,2,3, \ldots
\end{aligned}
$$

2. We call (3.13)/(3.14) the step-by-step Case sum rules.

Theorem 3.2 (One-Sided Step-by-Step Sum Rules). Let $J$ be a $B W$ matrix. $Z_{1}^{ \pm}(J)<\infty$ if and only if $Z_{1}^{ \pm}\left(J^{(1)}\right)<\infty$, and if $Z_{1}^{ \pm}(J)<\infty$, then we have for $\ell=1,3,5, \ldots$,

$$
Z_{\ell}^{ \pm}(J)=-\ln \left(a_{1}\right) \mp \frac{1}{2} \zeta_{\ell}^{(1)}(J)+X_{0}^{(1)}(J) \mp \frac{1}{2} X_{\ell}^{(1)}(J)+Z_{\ell}^{ \pm}\left(J^{(1)}\right)
$$

Remark. Theorem 3.2 is intended to be two statements: one with all the upper signs used and one with all the lower signs used.

Theorem 3.3 (Quasi-Step-by-Step Sum Rules). Let $J$ be a $B W$ matrix. $Z_{2}^{-}(J)<\infty$ if and only if $Z_{2}^{-}\left(J^{(1)}\right)<\infty$, and if $Z_{2}^{-}(J)<\infty$, then for $\ell=2,4, \ldots$, we have

$$
Z_{\ell}^{-}(J)=-\ln \left(a_{1}\right)+\frac{1}{2} \zeta_{\ell}^{(1)}(J)+X_{0}^{(1)}(J)+\frac{1}{2} X_{\ell}^{(1)}(J)+Z_{\ell}^{-}\left(J^{(1)}\right)
$$

Remarks. 1. The name comes from the fact that since $1-\cos 2 \theta=$ $2 \sin ^{2} \theta, Z_{2}^{-}(J)$ is what Killip-Simon [10 called the quasi-Szegö integral

$$
Z_{2}^{-}(J)=\frac{1}{2 \pi} \int_{0}^{2 \pi} \ln \left(\frac{\sin \theta}{\operatorname{Im} M\left(e^{i \theta}, J\right)}\right) \sin ^{2} \theta d \theta
$$

2. Since $Z(J)<\infty$ implies $Z_{1}^{+}(J)$ and $Z_{1}^{-}(J)<\infty$, and $Z_{1}^{+}(J)$ or $Z_{1}^{-}(J)<\infty$ imply $Z_{2}^{-}(J)<\infty$, we have additional sum rules in various cases.

3. In [11], Laptev et al. prove sum rules for $Z_{\ell}^{-}(J)$ where $\ell=$ $4,6,8, \ldots$ One can develop step-by-step sum rules in this case and use it to streamline the proof of their rules as we streamline the proof of the Killip-Simon $P_{2}$ rule (our $Z_{2}^{-}$sum rule) in the next section.

The step-by-step sum rules were introduced in Killip-Simon, who first take $r<1$ (in our language below), then take $n \rightarrow \infty$, and then $r \uparrow 1$ with some technical hurdles to take $r \uparrow 1$. By first letting $r \uparrow 1$ with $n<\infty$, and then $n \rightarrow \infty$ as in the next section, we can both 
simplify their proof and obtain additional results. The idea of using the imaginary part of

$$
-M(z ; J)^{-1}=-\left(z+z^{-1}\right)+b_{1}+a_{1}^{2} M\left(z ; J^{(1)}\right)
$$

is taken from Killip-Simon [10].

Proof of Theorem 3.1. Taking imaginary parts of both sides of (3.20) with $z=r e^{i \theta}$ and $r<1$, we obtain

$$
\left[\operatorname{Im} M\left(r e^{i \theta} ; J\right)\right]\left|M\left(r e^{i \theta} ; J\right)\right|^{-2}=\left(r^{-1}-r\right) \sin \theta+a_{1}^{2} \operatorname{Im} M\left(r e^{i \theta} ; J^{(1)}\right)
$$

Taking ln's of both sides, we obtain

$$
\ln \left(\frac{\sin \theta}{\operatorname{Im} M\left(r e^{i \theta} ; J\right)}\right)=t_{1}+t_{2}+t_{3}
$$

where

$$
\begin{aligned}
& t_{1}=-2 \ln \left|M\left(r e^{i \theta} ; J\right)\right| \\
& t_{2}=-2 \ln a_{1} \\
& t_{3}=\ln \left(\frac{\sin \theta}{g(r) \sin \theta+\operatorname{Im} M\left(r e^{i \theta} ; J^{(1)}\right)}\right)
\end{aligned}
$$

where

$$
g(r)=a_{1}^{-2}\left(r^{-1}-r\right)
$$

Let

$$
f(z)=\frac{M(r z ; J)}{r z}
$$

so $f(0)=1$ (see $(3.20))$. In the unit disk, $f(z)$ is meromorphic and has poles at $\left\{r\left(\beta_{j}^{ \pm}(J)\right)^{-1} \mid j\right.$ so that $\left.\beta_{j}^{ \pm}(J)>r^{-1}\right\}$ and zeros at $\left\{r\left(\beta_{j}^{ \pm}\left(J^{(1)}\right)\right)^{-1} \mid \beta_{j}^{ \pm}\left(J^{(1)}\right)>r^{-1}\right\}$. Thus, by Jensen's formula for $f$ :

$\frac{1}{4 \pi} \int_{0}^{2 \pi} t_{1} d \theta=-\ln r+\sum_{\beta_{j}^{ \pm}(J)>r^{-1}} \ln \left|r^{-1} \beta_{j}^{ \pm}(J)\right|-\sum_{\beta_{j}^{ \pm}\left(J^{(1)}\right)>r^{-1}} \ln \left|r^{-1} \beta_{j}^{ \pm}\left(J^{(1)}\right)\right|$

By (3.1), the number of terms in the sums differs by at most 2 , so that the $\ln \left(r^{-1}\right)$ 's cancel up to at most $2 \ln \left(r^{-1}\right) \rightarrow 0$ as $r \uparrow 1$. Thus as $r \uparrow 1$,

$$
\frac{1}{4 \pi} \int_{0}^{2 \pi}\left(t_{1}+t_{2}\right) d \theta \rightarrow-\ln \left(a_{1}\right)+X_{0}^{(1)}(J)
$$

It follows by $(3.22)$ and Theorems 2.1 and 2.8 (with $w(\varphi)=1$ ) that $Z(J)<\infty$ if and only if $Z\left(J^{(1)}\right)<\infty$, and if they are finite, (3.13) holds. 
It also follows that if $Z(J)<\infty$, we have $L^{1}$ convergence of the ln's to their $r=1$ values. That implies convergence of the integrals with $\cos (\ell \theta)$ inside. Higher Jensen's formula as in [10] then implies (3.14). In place of $\ln \left|\beta r^{-1}\right|$, we have $(r \beta)^{\ell}-(r \beta)^{-\ell}$, but the sums still converge to the $r=1$ limit since we can separate the $\beta^{\ell}$ and $\beta^{-\ell}$ terms, and then the $r$ 's factor out.

Proofs of Theorems 3.2 and 3.3. These are the same as the above proof, but now the weight $w$ is either $1 \pm \cos (\theta)$ or $1-\cos (2 \theta)$ and that weight obeys (2.3) and (2.4).

Corollary 3.4. Let $J$ be a $B W$ matrix. If $J$ and $\tilde{J}$ differ by a finite rank perturbation, then $J$ is Szegö (resp. Szegö at \pm 2 ) if and only if $\tilde{J}$ is.

Proof. For some $n, J^{(n)}=\tilde{J}^{(n)}$, so this is immediate from Theorems 3.1 and 3.2 .

Conjecture 3.5. Let $J$ be a BW matrix. If $J$ and $\tilde{J}$ differ by a trace class perturbation, then $J$ is Szegő (resp. Szegő at \pm 2 ) if and only if $\tilde{J}$ is. It is possible this conjecture is only generally true if $J-J_{0}$ is only assumed compact or is only assumed Hilbert-Schmidt.

This conjecture for $J=J_{0}$ is Nevai's conjecture recently proven by Killip-Simon. Their method of proof and the ideas here would prove this conjecture if one can prove a result of the following form. Let $J, \tilde{J}$ differ by a finite rank operator so that by the discussion before (3.2),

$$
\lim _{N \rightarrow \infty} \sum_{ \pm} \sum_{j=1}^{N}\left(\sqrt{E_{j}^{ \pm}(J)^{2}-4}-\sqrt{E_{j}^{ \pm}(\tilde{J})^{2}-4}\right) \equiv \delta(J, \tilde{J})
$$

exists and is finite. The conjecture would be provable by the method of [10] and this paper (by using the step-by-step sum rule to remove the first $m$ pieces of $J$ and then replacing them with the first $m$ pieces of $\tilde{J}$ ) if one had a bound of the form

$$
|\delta(J, \tilde{J})| \leq \text { (const.) } \operatorname{Tr}(|J-\tilde{J}|)
$$

(3.28) with $J=J_{0}$ is the estimate of Hundertmark-Simon 9]. We have counterexamples that show (3.28) does not hold for a universal constant $c$. However, in these examples, $\|J\| \rightarrow \infty$ as $c \rightarrow \infty$. Thus it could be that (3.28) holds with $c$ only depending on $J$ for some class of $J$ 's. If it held with a bound depending only on $\|J\|$, the conjecture would hold in general. If $J$ was required in $J_{0}+$ Hilbert-Schmidt, we would get the conjecture for such $J$ 's. 


\section{The $Z_{0}, Z_{1}^{ \pm}$, AND $Z_{2}^{-}$Sum Rules}

Our goal here is to prove that sum rules of Case type hold under certain hypotheses. Of interest on their own, these considerations also somewhat simplify the proof of the $P_{2}$ sum rule in Section 8 of [10], and considerably simplify the proof of the $C_{0}$ sum rule for trace class $J-J_{0}$ from Section 9 of [10]. Throughout, $J$ will be a BW matrix. There are two main tools. As in [10], lower semicontinuity of the $Z$ 's in $J$ (in the topology of pointwise convergence of matrix elements) gets inequalities in one direction. We use step-by-step sum rules and boundedness from below of $Z$ for the other direction.

We first introduce some quantities involving a fixed Jacobi matrix:

$$
\begin{aligned}
& \bar{A}_{0}(J)=\limsup _{n \rightarrow \infty}\left(-\sum_{j=1}^{n} \ln \left(a_{j}\right)\right) \\
& \underline{A}_{0}(J)=\liminf _{n \rightarrow \infty}\left(-\sum_{j=0}^{n} \ln \left(a_{j}\right)\right) \\
& \bar{A}_{1}^{ \pm}(J)=\limsup _{n \rightarrow \infty}\left(-\sum_{j=1}^{n}\left(a_{j}-1 \pm \frac{1}{2} b_{j}\right)\right) \\
& \underline{A}_{1}^{ \pm}(J)=\liminf _{n \rightarrow \infty}\left(-\sum_{j=1}^{n}\left(a_{j}-1 \pm \frac{1}{2} b_{j}\right)\right) \\
& A_{2}(J)=\sum_{j=1}^{\infty}\left[\frac{1}{4} b_{j}^{2}+\frac{1}{2} G\left(a_{j}\right)\right]
\end{aligned}
$$

where

$$
G(a)=a^{2}-1-\ln \left(a^{2}\right)
$$

Since $G(a) \geq 0$, the finite sums have a limit (which may be $+\infty$ ).

We note that for $a$ near $1, G(a) \sim 2(a-1)^{2}$. Thus $A_{2}(J)$ is finite if and only if $J-J_{0}$ is Hilbert-Schmidt. In (4.2), we can use $a_{j}-1$ in place of $\ln \left(a_{j}\right)$ because if $\left\{a_{j}-1\right\} \in \ell^{2}$ (e.g., if $J-J_{0}$ is Hilbert-Schmidt), then $\sum\left|\ln \left(a_{j}\right)-\left(a_{j}-1\right)\right|<\infty$.

We also introduce some functions of the eigenvalues:

$$
\begin{aligned}
\mathcal{E}_{0}(J) & =\sum_{j, \pm} \ln \left|\beta_{j}^{ \pm}\right| \\
\mathcal{E}_{1}^{ \pm}(J) & =\sum_{j} \sqrt{\left(E_{j}^{ \pm}\right)^{2}-4}
\end{aligned}
$$




$$
\mathcal{E}_{2}(J)=\sum_{j, \pm} F\left(E_{j}^{ \pm}\right)
$$

where $F(E)=\frac{1}{4}\left[\beta^{2}-\beta^{-2}-\ln \left(\beta^{4}\right)\right]$ with $E=\beta+\beta^{-1}$ and $|\beta|>1$. For $|E| \sim 2, F(E)$ is $O\left((|E|-2)^{3 / 2}\right)$. In $(4.4)$ and (4.6), we sum over + and - . In (4.5), we define $\mathcal{E}_{1}^{+}$and $\mathcal{E}_{1}^{-}$with only the + or only the terms.

We need the following basis-dependent notion:

Definition. Let $B$ be a bounded operator on $\ell^{2}\left(\mathbb{Z}^{+}\right)$. We say $B$ has a conditional trace if

$$
\lim _{\ell \rightarrow \infty} \sum_{j=1}^{\ell}\left\langle\delta_{j}, B \delta_{j}\right\rangle \equiv \mathrm{c}-\operatorname{Tr}(B)
$$

exists and is finite.

If $B$ is not trace class, this object is not unitarily invariant.

Our goal in this section is to prove the following theorems whose proof is deferred until after all the statements.

Theorem 4.1. Let $J$ be a $B W$ matrix. Consider the four statements:

(i) $\bar{A}_{0}(J)>-\infty$

(ii) $\underline{A}_{0}(J)<\infty$

(iii) $Z(J)<\infty$

(iv) $\mathcal{E}_{0}(J)<\infty$

Then

(a) (ii) + (iv) $\Rightarrow$ (iii) + (i)

(b) (i) + (iii) $\Rightarrow$ (iv) + (ii)

(c) (iii) $\Rightarrow \bar{A}_{0}(J)<\infty$

(d) (iv) $\Rightarrow \underline{A}_{0}(J)>-\infty$

Thus (iii) + (iv) $\Rightarrow$ (i) + (ii). In particular, if $\underline{A}_{0}(J)=\bar{A}_{0}(J)$, that $i s$, the limit exists, then the finiteness of any two of $Z(J), \mathcal{E}_{0}(J)$, and $\bar{A}_{0}(J)$ implies the finiteness of the third.

If all four conditions hold and $J-J_{0}$ is compact, then

(e)

$$
\lim _{n \rightarrow \infty}\left(-\sum_{j=1}^{n} \ln \left(a_{j}\right)\right) \equiv A_{0}(J)
$$

exists and is finite, and

$$
Z(J)=A_{0}(J)+\mathcal{E}_{0}(J)
$$

(f) For each $\ell=1,2, \ldots$,

$$
-\sum_{j, \pm} \ell^{-1}\left[\beta_{j}^{ \pm}(J)^{\ell}-\beta_{j}^{ \pm}(J)^{-\ell}\right] \equiv X_{\ell}^{(\infty)}(J)
$$


converges absolutely and equals $\lim _{n \rightarrow \infty} X_{\ell}^{(n)}(J)$.

(g) For each $\ell=1,2, \ldots$,

$$
B_{\ell}(J)=\frac{2}{\ell}\left\{T_{\ell}\left(\frac{J}{2}\right)-T_{\ell}\left(\frac{J_{0}}{2}\right)\right\}
$$

has a conditional trace and

$$
\mathrm{c}-\operatorname{Tr}\left(B_{\ell}(J)\right)=\lim _{n \rightarrow \infty} \zeta_{\ell}^{(n)}(J)
$$

for example, if $\ell=1, \sum_{j=1}^{n} b_{j}$ converges to a finite limit.

(h) The Case sum rule holds:

$$
Y_{\ell}(J)=\mathrm{c}-\operatorname{Tr}\left(B_{\ell}(J)\right)+X_{\ell}^{(\infty)}(J)
$$

where $Y_{\ell}$ is given by (3.11), $X_{\ell}^{(\infty)}$ by (4.10), and $\mathrm{c}-\operatorname{Tr}\left(B_{\ell}(J)\right)$ by (4.7), (4.11), and (4.12).

Remarks. 1. In one sense, this is the main result of this paper.

2. We will give examples later where $\bar{A}_{0}(J)=\underline{A}_{0}(J)$ and one of the conditions (i)/(ii), (iii), (iv) holds and the other two fail.

3. For $\ell$ odd, $T_{\ell}\left(J_{0} / 2\right)$ vanishes on-diagonal. By Proposition 2.2 of [10 and the fact that the diagonal matrix elements of $J_{0}^{k}$ are eventually constant, it follows that for $\ell$ even, $T_{\ell}\left(J_{0} / 2\right)$ eventually vanishes on-diagonal and $\mathrm{c}-\operatorname{Tr}\left(T_{\ell}\left(J_{0} / 2\right)\right)=-\frac{1}{2}$. Thus $(\mathrm{g})$ says $\mathrm{c}-\operatorname{Tr}\left(T_{\ell}(J / 2)\right)$ exists and the sum rule $(4.13)$ can replace $\mathrm{c}-\operatorname{Tr}\left(B_{\ell}(J)\right)$ by $\mathrm{c}-\operatorname{Tr}\left(T_{\ell}(J / 2)\right)$ plus a constant (zero if $\ell$ is odd and $1 / \ell$ if $\ell$ is even). For $\ell$ even, $\mathrm{c}-\operatorname{Tr}\left(T_{\ell}\left(J_{0} / 2\right)\right)=-\frac{1}{2}$ while $\operatorname{Tr}\left(T_{\ell}\left(J_{0, n ; F} / 2\right)\right)=-1$ for $n$ large because $T_{\ell}\left(J_{0, n ; F} / 2\right)$ has two ends.

Corollary 4.2. Let $J-J_{0}$ be compact. If $Z(J)<\infty$, then $-\sum_{j=1}^{n} \ln \left(a_{j}\right)$ either converges or diverges to $-\infty$.

Remarks. 1. We will give an example later where $Z(J)<\infty$, and $\lim _{n \rightarrow \infty}\left(-\sum_{j=1}^{n} \ln \left(a_{j}\right)\right)=-\infty$.

2. In other words, if $J-J_{0}$ is compact and $\bar{A}_{0}(J) \neq \underline{A}_{0}(J)$, then $Z(J)=\infty$.

3. Similarly, if $J-J_{0}$ is compact and $\mathcal{E}_{0}(J)<\infty$, then the limit exists and is finite or is $+\infty$.

Proof. If $Z(J)<\infty$ and $\bar{A}_{0}>-\infty$, then by (b) of the theorem, all four conditions hold, and so by (e), the limit exists. On the other hand, if $\bar{A}_{0}=-\infty$, then $\bar{A}_{0}=\underline{A}_{0}=-\infty$.

Corollary 4.3. If $J-J_{0}$ is trace class, then $Z(J)<\infty, \mathcal{E}_{0}(J)<\infty$, and the sum rules (4.9) and (4.13) hold. 
Remark. This is a result of Killip-Simon [10]. Our proof that $Z(J)<$ $\infty$ is essentially the same as theirs, but our proof of the sum rules is much easier.

Proof. Since $J-J_{0}$ is trace class, it is compact. Clearly, $\bar{A}_{0}=\underline{A}_{0}$, and is neither $\infty$ nor $-\infty$ since $a_{j}>0$ and $\sum\left|a_{j}-1\right|<\infty$ imply $\sum\left|\ln \left(a_{j}\right)\right|<\infty$. By the bound of Hundertmark-Simon [9], $\mathcal{E}_{0}(J)<\infty$. The sum rules then hold by (a), (e), and (h) of Theorem 4.1.

Theorem 4.4. Suppose $J-J_{0}$ is Hilbert-Schmidt. Then

(i) $\underline{A}_{1}^{ \pm}<\infty$ and $\mathcal{E}_{1}^{ \pm}<\infty$ implies $Z_{1}^{ \pm}<\infty$.

(ii) $Z_{1}^{ \pm}<\infty$ implies $\bar{A}_{1}^{ \pm}<\infty$.

(iii) $Z_{1}^{ \pm}<\infty$ and $\bar{A}_{1}^{ \pm}>-\infty$ implies $\mathcal{E}_{1}^{ \pm}<\infty$.

(iv) $\mathcal{E}_{1}^{ \pm}<\infty$ implies $\underline{A}_{1}^{ \pm}>-\infty$.

Remarks. 1. Each of (i)-(iv) is intended as two statements.

2. In Section 6, we will explore (ii), which is the most striking of these results since its contrapositive gives very general conditions under which the Szegő condition fails.

3. The Hilbert-Schmidt condition in (i) and (iv) can be replaced by the somewhat weaker condition that

$$
\sum_{j, \pm}\left(\left|E_{j}^{ \pm}\right|-2\right)^{3 / 2}<\infty
$$

That is true for (ii) and (iii) also, but by the $Z_{2}^{-}$sum rule, (4.14) plus $Z_{1}^{ \pm}<\infty$ implies $J-J_{0}$ is Hilbert-Schmidt.

Theorem 4.5. Let $J$ be a $B W$ matrix. Then

$$
Z_{2}^{-}(J)+\mathcal{E}_{2}(J)=A_{2}(J)
$$

Remarks. 1. This is, of course, the $P_{2}$ sum rule of Killip-Simon 10. Our proof that $Z_{2}^{-}(J)+\mathcal{E}_{2}(J) \leq A_{2}(J)$ is identical to that in [10], but our proof of the other half is somewhat streamlined.

2. As in [10], the values $+\infty$ are allowed in (4.15).

Proof of Theorem 4.1. As in [10], let $J_{n}$ be the infinite Jacobi matrix obtained from $J$ by replacing $a_{\ell}$ by 1 if $\ell \geq n$ and $b_{\ell}$ by 0 if $\ell \geq n+1$. Then (3.15) (noting $J_{n}^{(n)}=J_{0}$ and $Z\left(J_{0}\right)=0$ ) reads

$$
Z\left(J_{n}\right)=-\sum_{j=1}^{n} \ln \left(a_{j}\right)+\sum_{j, \pm} \ln \left|\beta_{j}^{ \pm}\left(J_{n}\right)\right|
$$


[10, Section 6] implies the eigenvalue sum converges to $\mathcal{E}_{0}(J)$ if $J-J_{0}$ is compact, and in any event, is bounded above by $\mathcal{E}_{0}(J)+c_{0}$ where $c_{0}=0$ if $J-J_{0}$ is compact and otherwise,

$$
c_{0}=\ln \left|\beta_{1}^{+}(J)\right|+\ln \left|\beta_{1}^{-}(J)\right|
$$

Moreover, by semicontinuity of the entropy [10, Section 5], $Z(J) \leq$ $\lim \inf Z\left(J_{n}\right)$. Thus we have

$$
Z(J) \leq \underline{A}_{0}(J)+\mathcal{E}_{0}(J)+c_{0}
$$

Thus far, the proof is directly from [10]. On the other hand, by (3.15), we have

$$
Z(J) \geq \bar{A}_{0}(J)+\liminf X_{0}^{(n)}(J)+\liminf Z\left(J^{(n)}\right)
$$

By the lemma below, $\lim _{n \rightarrow \infty} X_{0}^{(n)}(J)=\mathcal{E}_{0}(J)$. Moreover, by Theorem 5.5 (eqn. (5.26)) of Killip-Simon [10], $Z\left(J^{(n)}\right) \geq-\frac{1}{2} \ln (2)$, and if $J^{(n)} \rightarrow J_{0}$ in norm, that is, $J-J_{0}$ is compact, then by semicontinuity of $Z, 0=Z\left(J_{0}\right) \leq \liminf Z\left(J^{(n)}\right)$. Therefore, (4.19) implies that

$$
Z(J) \geq \bar{A}_{0}(J)+\mathcal{E}_{0}(J)-c
$$

where

$$
c=0 \text { if } J-J_{0} \text { is compact; } \quad c=\frac{1}{2} \ln 2 \text { in general }
$$

With these preliminaries out of the way,

Proof of (d). (iv) and (4.18) imply that

$$
\bar{A}_{0}(J) \geq \underline{A}_{0}(J) \geq Z(J)-\mathcal{E}_{0}(J)-c_{0}>-\infty
$$

Proof of (a). (4.18) shows $Z(J)<\infty$, and (d) shows that (i) holds.

Proof of (c). By (4.20) and $\mathcal{E}_{0}(J) \geq 0$,

$$
Z(J) \geq \bar{A}_{0}(J)-c
$$

so $Z(J)<\infty$ implies $\bar{A}_{0}(J)<\infty$.

Proof of (b). Since $\bar{A}_{0}(J)>-\infty$ and $c<\infty$, (4.20) plus $Z(J)<\infty$ implies $\mathcal{E}_{0}(J)<\infty$. (c) shows that (ii) holds.

Note that (iii), (iv), and (4.20) imply that

$$
\underline{A}_{0}(J) \leq \bar{A}_{0}(J) \leq Z(J)-\mathcal{E}_{0}(J)+\frac{1}{2} \ln 2<\infty
$$

Thus we have shown more than merely (iii) + (iv) $\Rightarrow$ (i) + (ii), namely, (iii) + (iv) imply by (4.22) and (4.23)

$$
-\infty<\bar{A}_{0}(J) \leq \underline{A}_{0}(J)+\frac{1}{2} \ln 2+c_{0}<\infty
$$

We can say more if $J-J_{0}$ is compact. 
Proof of (e). (4.23) is now replaced by

$$
\underline{A}_{0}(J) \leq \bar{A}_{0}(J) \leq Z(J)-\mathcal{E}_{0}(J)
$$

since we can take $c=0$ in (4.20). This plus (4.22) with $c_{0}=0$ implies $\bar{A}_{0}(J)=\underline{A}_{0}(J)$ and $(4.9)$.

Proof of (f), (g), (h). We have the sum rules (3.15), (3.16). $Z(J) \pm$

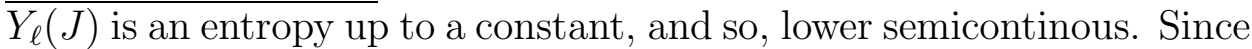
$\left\|J^{(n)}-J_{0}\right\| \rightarrow 0$, we have

$$
\liminf \left(Z\left(J^{(n)}\right) \pm Y_{\ell}\left(J^{(n)}\right)\right) \geq 0
$$

On the other hand, since $Z\left(J^{(n)}\right)<\infty$ and $\mathcal{E}_{0}\left(J^{(n)}\right) \leq \mathcal{E}_{0}(J)<\infty$, $J^{(n)}$ obeys the sum rule (4.9). Since $-\sum_{j=1}^{n} \ln \left(a_{j}\right)$ converges conditionally

$$
\lim _{n \rightarrow \infty} \lim _{m \rightarrow \infty}\left(-\sum_{j=n}^{m+n} \ln \left(a_{j}\right)\right)=0
$$

Moreover, $\mathcal{E}_{0}\left(J^{(n)}\right) \rightarrow 0$ by Lemma 4.6 below and we conclude that $\lim Z\left(J^{(n)}\right)=0$. Thus (4.25) becomes

$$
\liminf _{n} Y_{\ell}\left(J^{(n)}\right) \geq 0, \quad \limsup _{n} Y_{\ell}\left(J^{(n)}\right) \leq 0
$$

or

$$
\lim _{n} Y_{\ell}\left(J^{(n)}\right)=0
$$

By the lemma below, $\lim _{n} X_{\ell}^{(n)}(J)=X_{\ell}^{(\infty)}(J)$ exists and is finite. Since $\mathcal{E}_{0}(J)<\infty$, we have that the sum defining $X_{\ell}^{(\infty)}(J)$ is absolutely convergent. This proves (f).

By this fact, (3.16), and (4.26), $\lim _{n \rightarrow \infty} \zeta_{\ell}^{(n)}(J)$ exists, is finite, and obeys the sum rule

$$
Y_{\ell}(J)=\lim _{n \rightarrow \infty} \zeta_{\ell}^{(n)}(J)+X_{\ell}^{(\infty)}(J)
$$

By Propositions 2.2 and 4.3 of Killip-Simon [10], the existence of $\lim _{n \rightarrow \infty} \zeta_{\ell}^{(n)}(J)$ is precisely the existence of the conditional trace.

Lemma 4.6. Let $J$ be a $B W$ matrix. Let $f$ be a monotone increasing continuous function on $[2, \infty)$ with $f(2)=0$. Then

$$
\lim _{n \rightarrow \infty} \sum_{j=1}^{\infty}\left[f\left(E_{j}^{+}(J)\right)-f\left(E_{j}^{+}\left(J^{(n)}\right)\right)\right]=\sum_{j=1}^{\infty} f\left(E_{j}^{+}(J)\right)
$$


Remarks. 1. The right side of (4.27) may be finite or infinite.

2. The sum on the left is interpreted as the limit of the sum from 1 to $n$ as $n \rightarrow \infty$, which exists and is finite by the arguments at the start of Section 3.

3. A similar result holds for $E_{j}^{-}$and $f$ monotone decreasing on $(-\infty,-2]$.

Proof. Call the sum on the left of 4.27$)(\delta f)(J, n)$. Since $E_{j}^{+}\left(J^{(n)}\right) \leq$ $E_{j}^{+}(J)$, we have

$$
(\delta f)(J, n) \geq \sum_{j=1}^{m}\left[f\left(E_{j}^{+}(J)\right)-f\left(E_{j}^{+}\left(J^{(n)}\right)\right)\right]
$$

so, if we show for each fixed $j$ as $n \rightarrow \infty$,

$$
E_{j}^{+}\left(J^{(n)}\right) \rightarrow 2
$$

we have, by taking $n \rightarrow \infty$ and then $m \rightarrow \infty$, that

$$
\liminf (\delta f)(J, n) \geq \sum_{j=1}^{\infty} f\left(E_{j}^{+}(J)\right)
$$

On the other hand, since $f \geq 0$, for each $m$,

$$
\sum_{j=1}^{m}\left[f\left(E_{j}^{+}(J)\right)-f\left(E_{j}^{+}\left(J^{(n)}\right)\right)\right] \leq \sum_{j=1}^{m} f\left(E_{j}^{+}(J)\right)
$$

so taking $m$ to infinity and then $n \rightarrow \infty$,

$$
\lim \sup (\delta f)(J, n) \leq \sum_{j=1}^{\infty} f\left(E_{j}^{+}(J)\right)
$$

Thus (4.29) implies the result, so we need only prove that.

Fix $\varepsilon>0$ and look at the solution of the orthogonal polynomial sequence $u_{n}=P_{n}(2+\varepsilon)$ as a function of $n$. By Sturm oscillation theory [7], the number of sign changes of $u_{n}$ (i.e., number of zeros of the piecewise linear interpolation of $u_{n}$ ) is the number of $j$ with $E_{j}^{+}(J)>2+\varepsilon$. Since $J$ is a BW matrix, this is finite, so there exist $N_{0}$ with $u_{n}$ of definite sign if $n \geq N_{0}-1$. It follows by Sturm oscillation theory again that for all $j$,

$$
E_{j}^{+}\left(J^{(n)}\right) \leq 2+\varepsilon
$$

if $n \geq N_{0}$. This implies (4.29). 
The combination of this Sturm oscillation argument and Theorem 3.1 gives one tools to handle finitely many bound states as an alternate to Nikishin [15]. For the oscillation argument says that if $J$ has finitely many eigenvalues outside $[-2,2]$, there is a $J^{(n)}$ with no eigenvalues. On the other hand, by Theorem 3.1, $Z(J)<\infty$ if and only if $Z\left(J^{(n)}\right)<\infty$.

Proof of Theorem 4.5. $Z_{2}^{-}(J)$ is an entropy and not merely an entropy up to a constant (see [10]). Thus $Z_{2}^{-}\left(J^{(n)}\right) \geq 0$ for all $J^{(n)}$. Moreover, since the terms in $A_{2}$ are positive, the limit exists. Thus, following the proofs of (4.18) and (4.20) but using (3.18) in place of (3.15),

$$
Z_{2}^{-}(J)+\mathcal{E}_{2}(J) \leq A_{2}(J)
$$

and

$$
Z_{2}^{-}(J)+\mathcal{E}_{2}(J) \geq A_{2}(J)
$$

which yields the $P_{2}$ sum rule. In the above, we use the fact that in place of $Z(J) \geq-\frac{1}{2} \ln (2)$, one has $Z_{2}^{-}(J) \geq 0$, and the fact that $A_{2}(J)<\infty$ implies that $J-J_{0}$ is compact.

Proof of Theorem 4.4. Let $g(\beta)=\ln \beta-\frac{1}{2}\left(\beta-\beta^{-1}\right)$ in the region $\beta>0$. Then

$$
g^{\prime}(\beta)=\beta^{-1}-\frac{1}{2}-\frac{1}{2} \beta^{-2}=-\frac{1}{2} \beta^{-2}(\beta-1)^{2}
$$

so $g$ is analytic near $\beta=1$ and $g(1)=g^{\prime}(1)=g^{\prime \prime}(1)=0$, that is, $g(\beta) \sim c(\beta-1)^{3}$. On the other hand, $h(\beta)=\ln \beta+\frac{1}{2}\left(\beta-\beta^{-1}\right)$ is $g(\beta)+\left(\beta-\beta^{-1}\right)=\beta-\beta^{-1}+O\left((\beta-1)^{3}\right)$. Since $\beta+\beta^{-1}=E$ means $\beta-\beta^{-1}=\sqrt{E^{2}-4}$ and $\beta-1=O(\sqrt{E-2})$, we conclude that

$$
\begin{aligned}
E>2 \Rightarrow \ln (\beta)-\frac{1}{2}\left(\beta-\beta^{-1}\right) & =O\left(|E-2|^{3 / 2}\right) \\
\ln (\beta)+\frac{1}{2}\left(\beta-\beta^{-1}\right) & =\sqrt{E^{2}-4}+O\left(|E-2|^{3 / 2}\right)
\end{aligned}
$$

while

$$
\begin{aligned}
E<-2 \Rightarrow \ln (|\beta|)-\frac{1}{2}\left(\beta-\beta^{-1}\right) & =\sqrt{E^{2}-4}+O\left(|E+2|^{3 / 2}\right) \\
\ln (|\beta|)+\frac{1}{2}\left(\beta-\beta^{-1}\right) & =O\left(|E+2|^{3 / 2}\right)
\end{aligned}
$$

It follows, using Lemma 4.6, that

$$
\lim _{n \rightarrow \infty} X_{0}^{(n)}(J) \mp \frac{1}{2} X_{1}^{(n)}(J)=\mathcal{E}_{1}^{ \pm}+\text {bdd }
$$

since Theorem 4.5 implies $\sum_{j, \pm}\left(\sqrt{E_{j}^{ \pm 2}-4}\right)^{3}<\infty$ (or, by results of [9]). Thus for a constant $c_{1}$ dependng only on $\left\|J-J_{0}\right\|_{2}$, we have

$$
Z_{1}^{ \pm}(J) \leq c_{1}+\underline{A}_{1}^{ \pm}+\mathcal{E}_{1}^{ \pm}
$$


by writing the finite rank sum rule, taking limits and using the argument between (4.16) and (4.17). Since $Z_{1}^{ \pm}(J)$ are entropies up to a constant, we have $Z_{1}^{ \pm}\left(J^{(n)}\right) \geq-c_{2}$ and so by (3.17),

$$
Z_{1}^{ \pm}(J) \geq-c_{2}+\bar{A}_{1}^{ \pm}+\mathcal{E}_{1}^{ \pm}-c\left\|J-J_{0}\right\|_{2}^{2}
$$

With these preliminaries, we have

Proof of (i), (iv). Immediate from (4.32).

Proof of (ii). Since $\mathcal{E}_{1}^{ \pm} \geq 0$, 4.33) implies

$$
Z_{1}^{ \pm}(J) \geq-c_{2}+\bar{A}_{1}^{ \pm}
$$

so (ii) holds.

Proof of (iii). Immediate from (4.33).

Remark. (i)-(iv) of Theorem 4.4 are exactly (a)-(d) of Theorem 4.1 for the $Z_{1}^{ \pm}$sum rules. One therefore expects a version of (e) of that theorem to hold as well. Indeed, a modification of the above proof

yields for $J-J_{0}$ Hilbert-Schmidt that if $\mathcal{E}_{1}^{+}, Z_{1}^{+}, \bar{A}_{1}^{+}$are finite, then

$$
Z_{1}^{+}(J)=-\sum_{n=1}^{\infty}\left[\ln \left(a_{n}\right)+\frac{1}{2} b_{n}\right]+\sum_{j, \pm}\left[\ln \left|\beta_{j}^{ \pm}\right|+\frac{1}{2}\left(\beta_{j}^{ \pm}-\left(\beta_{j}^{ \pm}\right)^{-1}\right)\right]
$$

and if $\mathcal{E}_{1}^{-}, Z_{1}^{-}, \bar{A}_{1}^{-}$are finite, then

$$
Z_{1}^{-}(J)=-\sum_{n=1}^{\infty}\left[\ln \left(a_{n}\right)-\frac{1}{2} b_{n}\right]+\sum_{j, \pm}\left[\ln \left|\beta_{j}^{ \pm}\right|-\frac{1}{2}\left(\beta_{j}^{ \pm}-\left(\beta_{j}^{ \pm}\right)^{-1}\right)\right]
$$

\section{Shohat's Theorem With an Eigenvalue Estimate}

Shohat 21] translated Szegö's theory from the unit circle to the real line and was able to identify all Jacobi matrices which lead to measures with no mass points outside $[-2,2]$ and have $Z(J)<\infty$. The strongest result we know of this type is the following (Theorem $4^{\prime}$ ) from KillipSimon [10] (the methods of Nevai [13] can prove the same result):

Theorem 5.1. Let $\sigma(J) \subset[-2,2]$. Consider

(i) $\underline{A}_{0}(J)<\infty$ where $\underline{A}_{0}$ is given by (4.1).

(ii) $Z(J)<\infty$

(iii) $\sum_{n=1}^{\infty}\left(a_{n}-1\right)^{2}+\sum_{n=1}^{\infty} b_{n}^{2}<\infty$

(iv) $\underline{A}_{0}=\bar{A}_{0}$ and is finite.

(v) $\lim _{N \rightarrow \infty} \sum_{n=1}^{N} b_{n}$ exists and is finite. 
Then (under $\sigma(J) \subset[-2,2])$, we have

$$
\text { (i) } \Longleftrightarrow \text { (ii) }
$$

and either one implies (iii), (iv), and (v).

We can prove the following extension of this result:

Theorem 5.2. Theorem 5.1 remains true if $\sigma(J) \subset[-2,2]$ is replaced by $\sigma_{\text {ess }}(J) \subset[-2,2]$ and $(1.6)$.

Remarks. 1. Gončar [8], Nevai [13], and Nikishin [15] extended Shohat-type theorems to allow finitely many bound states outside $[-2,2]$.

2. Peherstorfer-Yuditskii [16] recently proved that $\mathcal{E}_{0}(J)<\infty$ and (ii) implies (iv) and additional results on polynomial asymptotics.

Proof. Let us suppose first $\sigma_{\text {ess }}(J)=[-2,2]$, so $J$ is a BW matrix. By Theorem 4.1(a), (i) of this theorem plus $\mathcal{E}_{0}(J)<\infty$ implies (ii) of this theorem. By Theorem 4.1(c), (ii) of this theorem implies (i) of this theorem.

If either holds, then (iv) follows from (e) of Theorem 4.1, (v) from the $\ell=1$ case of $(\mathrm{g})$ of Theorem 4.1. (iii) follows from Theorem 4.5 if we note that $\mathcal{E}_{0}<\infty$ implies $\mathcal{E}_{2}<\infty$, that $Z(J)<\infty$ implies $Z_{2}^{-}(J)<\infty$ and that $G(a)=O\left((a-1)^{2}\right)$.

If we only have a priori that $\sigma_{\text {ess }}(J) \subset[-2,2]$, we proceed as follows. If $Z(J)<\infty, \sigma_{\mathrm{ac}}(J) \supset[-2,2]$ so, in fact, $\sigma_{\mathrm{ess}}(J)=[-2,2]$. If $\underline{A}_{0}<\infty$, we look closely at the proof of Theorem 4.1.(a). (4.18) does not require $\sigma_{\text {ess }}(J)=[-2,2]$, but only that $\sigma_{\text {ess }}(J) \subset[-2,2]$. Thus, $\underline{A}_{0}<\infty$ implies $Z(J)<\infty$ if $\mathcal{E}_{0}(J)<\infty$.

There is an interesting way of rephrasing this. Let the normalized orthogonal polynomial obey

$$
P_{n}(x)=\gamma_{n} x^{n}+O\left(x^{n-1}\right)
$$

As is well known (see, e.g. 222),

$$
\gamma_{n}=\left(a_{1} a_{2} \ldots a_{n}\right)^{-1}
$$

Thus

$$
\underline{A}_{0}=\liminf \ln \left(\gamma_{n}\right)
$$

and

$$
\bar{A}_{0}=\limsup \ln \left(\gamma_{n}\right)
$$

Corollary 5.3. Suppose $\sigma_{\mathrm{ess}}(J) \subset[-2,2]$ and $\mathcal{E}_{0}(J)<\infty$. Then $Z(J)<\infty$ (i.e., the Szego" condition holds) if and only if $\gamma_{n}$ is bounded from above (and in that case, it is also bounded away from 0 ; indeed, $\lim \gamma_{n}$ exists and is in $\left.(0, \infty)\right)$. 
Remark. Actually, $\lim \sup \gamma_{n}<\infty$ is not needed; $\lim \inf \gamma_{n}<\infty$ is enough.

Proof. By (5.3), $\gamma_{n}$ bounded implies $\underline{A}_{0}<\infty$, and thus $Z(J)<\infty$. Conversely, $Z(J)<\infty$ implies $-\infty<\underline{A}_{0}=\bar{A}_{0}<\infty$. So by (5.2), it implies $\gamma_{n}$ is bounded above and below.

In the case of orthogonal polynomials on the circle, Szegö's theorem says $Z<\infty$ if and only if $\kappa_{j}$ is bounded if and only if $\sum_{j=1}^{\infty}\left|\alpha_{j}\right|^{2}<\infty$ where $\kappa_{j}$ is the leading coefficient of the normalized polynomials, and $\alpha_{j}$ are the Verblunsky (aka Geronimus, aka reflection) coefficients. In the real line case, if one drops the a priori requirement that $\mathcal{E}_{0}(J)<\infty$, it can happen that $\gamma_{n}$ is bounded but $Z(J)=\infty$. For example, if $a_{n} \equiv 1$ but $b_{n}=n^{-1}$, then $Z(J)$ cannot be finite. For $J-J_{0} \in \ell_{2}$, so Theorem 4.4(ii) is applicable and thus, $\bar{A}_{1}^{-}=\infty$ implies $Z(J)=\infty$.

But the other direction always holds:

Theorem 5.4. Let $J$ be a $B W$ matrix with $Z(J)<\infty$ (i.e., the Szegö condition holds). Then $\gamma_{n}$ is bounded. Moreover, if $J-J_{0}$ is compact, then $\lim _{n \rightarrow \infty} \gamma_{n}$ exists.

Remarks. 1. The examples of the next section show $Z(J)<\infty$ is consistent with $\lim \gamma_{n}=0$.

2. This result - even without a compactness hypothesis - is known. For $\gamma_{n}$ is monotone increasing in the measure (see, e.g., Nevai [14]) and so one can reduce to the case where Shohat's theorem applies.

Proof. By Theorem 4.1(c), $Z(J)<\infty$ implies $\bar{A}_{0}<\infty$ which, by (5.4), implies $\gamma_{n}$ is bounded. If $J-J_{0}$ is compact, then Corollary 4.2 implies that $\lim \gamma_{n}=\exp \left(\lim -\sum_{j=1}^{m} \ln \left(a_{j}\right)\right)$ exists but can be zero.

Here is another interesting application of Theorem 5.2.

Theorem 5.5. Suppose $b_{n} \geq 0$ and

$$
\sum_{n=1}^{\infty}\left|a_{n}-1\right|<\infty
$$

Then $\mathcal{E}_{0}(J)<\infty$ if and only if $\sum_{n=1}^{\infty} b_{n}<\infty$.

Proof. If $\sum_{n=1}^{\infty} b_{n}<\infty, \mathcal{E}_{0}(J)<\infty$ by (5.5) and the bounds of HundertmarkSimon [9]. On the other hand, if $\mathcal{E}_{0}(J)<\infty$, (5.5) implies $\underline{A}_{0}<\infty$, so by Theorem 5.2, $\sum_{n=1}^{N} b_{n}$ is convergent. Since $b_{n} \geq 0, \sum_{n=1}^{\infty} b_{n}<$ $\infty$. 


\section{6. $O\left(n^{-1}\right)$ Perturbations}

In this section, we will discuss examples where

$$
\begin{aligned}
& a_{n}=1+\alpha n^{-1}+E_{a}(n) \\
& b_{n}=\beta n^{-1}+E_{b}(n)
\end{aligned}
$$

where $E .(n)$ is small compared to $\frac{1}{n}$ in some sense. Our main result will involve the very weak requirement on the errors that $n\left(\left|E_{a}(n)\right|+\right.$ $\left.\left|E_{b}(n)\right|\right) \rightarrow 0$. (In fact, we only need the weaker condition that $\sum_{j=1}^{n}\left(\left|E_{a}(j)\right|+\right.$ $\left.\left|E_{b}(j)\right|\right)$ is $o(\ln n)$.) In discussing the historical context, we will consider stronger assumptions like

$$
E .(n)=\frac{\gamma}{n^{2}}+o\left(\frac{1}{n^{2}}\right)
$$

We will also mention examples where the leading $n^{-1}$ terms are replaced by $(-1)^{n} n^{-1}$.

These examples are natural because they are just at the borderline beyond $J-J_{0}$ trace class or $\underline{A}_{0}(J)<\infty$ or $\bar{A}_{0}(J)>-\infty$.

Here is the general picture for these examples. The $(\alpha, \beta)$ plane is divided into four regions:

(a) $|\beta|<-2 \alpha$. Szegö fails at both -2 and 2 .

(b) $|\beta| \leq 2 \alpha$. Szegö holds.

(c) $\beta>2|\alpha|$ or $\beta=-2 \alpha$ with $\beta>0$. Szego" holds at +2 but fails at -2 .

(d) $\beta<-2|\alpha|$ or $\beta=2 \alpha$ with $\beta<0$. Szegő holds at -2 but fails at +2 .

Remarks. 1 . These are only guidelines and the actual result that we can prove requires estimates on the errors.

2. Put more succinctly, Szegö holds at \pm 2 if and only if $2 \alpha \pm \beta \geq 0$.

3. We need strong hypotheses at the edges of our regions where $|\beta|=2|\alpha|$. For example, "generally" Szego" should hold if $\beta=2 \alpha>0$, but if $a_{n}=1+\alpha n^{-1}-(n \ln (n))^{-1}$ and $b_{n}=2 \alpha n^{-1}$, the Szegö condition fails (at -2 ), as follows from Theorem 6.1 below.

Here is the history of these kinds of problems:

(1) Pollaczek [17, 18, 19] found an explicit class of orthogonal polynomials in the region (in our language) $|\beta|<-2 \alpha$, one example for each such $(\alpha, \beta)$ with further study by Szego" [23, 25] (but note formula (1.7) in the appendix to Szegö's book [25] is wrong - he uses in that formula the Bateman project normalization of the parameters he calls $a, b$, not the normalization he uses elsewhere). They found that for these polynomials, the Szegö condition fails. 
(2) In [12], Nevai reported a conjecture of Askey that (with $O\left(n^{-2}\right)$ errors) Szegö fails for all $(\alpha, \beta) \neq(0,0)$.

(3) In [1], Askey-Ismail found some explicit examples with $b_{n} \equiv 0$ and $\alpha>0$, and noted that the Szegö condition holds (!), so they concluded the conjecture needed to be modified.

(4) In [6], Dombrowski-Nevai proved a general result that Szegő holds when $b_{n} \equiv 0$ and $\alpha>0$ with errors of the form (6.3).

(5) In [3], Charris-Ismail computed the weights for Pollaczek-type examples in the entire $(\alpha, \beta)$ plane to the left of the line $\alpha=1$, and considered a class depending on an additional parameter, $\lambda$. While they did not note the consequence for the Szegö condition, their example is consistent with our picture above.

In addition, we note that in [12], Nevai proved that the Szegö condition holds if $a_{n}=1+(-1)^{n} \alpha / n+O\left(n^{-2}\right)$ and $b_{n}=(-1)^{n} \beta / n+O\left(n^{-2}\right)$.

With regard to this class, here is our result in this paper:

Theorem 6.1. Suppose

$$
\begin{gathered}
\sum_{n=1}^{\infty}\left(a_{n}-1\right)^{2}+b_{n}^{2}<\infty \\
\limsup _{N}\left(-\sum_{n=1}^{N}\left(a_{n}-1 \pm \frac{1}{2} b_{n}\right)\right)=\infty
\end{gathered}
$$

for either plus or minus. Then the Szegö condition fails at \pm 2 .

Proof. (6.5) implies that $\bar{A}_{1}^{ \pm}(J)=\infty$ so by Theorem 4.4(ii), $Z_{1}^{ \pm}(J)=$ $\infty$.

Remark. The same kind of argument lets us also prove the failure of the Szegö conditoin without assuming (6.4) by replacing (6.5) by the slightly stronger condition that

$$
\limsup _{N}\left(-\sum_{n=1}^{N}\left(\ln \left(a_{n}\right) \pm \frac{1}{p} b_{n}\right)\right)=\infty
$$

for some $p>2$. For one can use the step-by-step sum rule for $(1 \pm$ $\left.\frac{2}{p} \cos (\theta)\right)$. (6.4) is not needed to control errors in $\mathcal{E}$-sums since they have a definite sign near both +2 and -2 , and it is not needed to replace $\ln (a)$ by $a-1$ since (6.6) has $\ln \left(a_{n}\right)$.

These considerations yield another interesting result. One can prove Theorem 4.1 for the weight $w(\theta)=1 \pm \frac{2}{p} \cos (\theta)$ just as we did it for the weight 1 . Since $w(\theta)$ is bounded away from zero, the corresponding $Z^{ \pm}$ term is finite if only if $Z$ is. Since $p>2$, the corresponding eigenvalue 
term is finite if and only if $\mathcal{E}_{0}$ is. Using Theorem 4.1(a)-(d) for this $w(\theta)$, we obtain

Theorem 6.2. Let $|p|<\frac{1}{2}$ and $|q|<\frac{1}{2}$.

(i) If

$$
\limsup _{N}\left(-\sum_{n=1}^{N}\left(\ln \left(a_{n}\right)+p b_{n}\right)\right)>-\infty
$$

and

$$
\limsup _{N}\left(-\sum_{n=1}^{N}\left(\ln \left(a_{n}\right)+q b_{n}\right)\right)=-\infty
$$

then $Z(J)=\infty$.

(ii) If

$$
\liminf _{N}\left(-\sum_{n=1}^{N}\left(\ln \left(a_{n}\right)+p b_{n}\right)\right)<\infty
$$

and

$$
\liminf _{N}\left(-\sum_{n=1}^{N}\left(\ln \left(a_{n}\right)+q b_{n}\right)\right)=\infty
$$

then $\mathcal{E}_{0}(J)=\infty$.

In particular, if $a_{n}=1, b_{n} \geq 0$, and $\sum_{n=1}^{\infty} b_{n}=\infty$, we have $Z(J)=$ $\infty$ and $\mathcal{E}_{0}(J)=\infty$. On the other hand, if instead $\sum_{n=1}^{\infty} b_{n}<\infty$, then $Z(J)<\infty$ and $\mathcal{E}_{0}(J)<\infty$ (see [10, 9]).

Corollary 6.3. If $a_{n}, b_{n}$ are given by (6.1), (6.2) with

$$
\lim _{n \rightarrow \infty} n\left[\left|E_{a}(n)\right|+\left|E_{b}(n)\right|\right]=0
$$

and $2 \alpha \pm \beta<0$, then the Szegö condition fails at \pm 2 .

Remarks. 1. This is intended as separate results for + and for - .

2. All we need is

$$
\lim _{n \rightarrow \infty}(\ln N)^{-1} \sum_{n=1}^{N}\left(\left|E_{a}(n)\right|+\left|E_{b}(n)\right|\right)=0
$$

instead of (6.7). In particular, trace class errors can be accommodated. Proof. If (6.7) holds,

$$
\sum_{n=1}^{N}\left(a_{n}-1\right) \pm \frac{1}{2} b_{n}=\left(\alpha \pm \frac{1}{2} \beta\right) \ln N+o(\ln N)
$$

so (6.5) holds if $2 \alpha \pm \beta<0$. 
As for the complementary region $|\beta| \leq 2 \alpha$, one of us has proven (see Zlatoš [27]) the following:

Theorem 6.4 (Zlatoš [27]). Suppose $|\beta| \leq 2 \alpha$ and

$$
\begin{aligned}
& a_{n}=1+\alpha n^{-1}+O\left(n^{-1-\varepsilon}\right) \\
& b_{n}=\beta n^{-1}+O\left(n^{-1-\varepsilon}\right)
\end{aligned}
$$

for some $\varepsilon>0$. Then the Szegö condition holds.

Remarks. 1. This is a corollary of a more general result (see [27]).

2. In these cases, $-\sum_{n=1}^{N} \ln \left(a_{n}\right)$ diverges to $-\infty$. This is only consistent with (4.18) because $\mathcal{E}_{0}(J)=\infty$, that is, the eigenvalue sum diverges and the two infinities cancel.

We can use these examples to illustrate the limits of Theorem 4.1:

(1) If $a_{n}=1$ and $b_{n}=\frac{1}{n}$, then $Z(J)=\infty$ (by Corollary 6.3) while $\bar{A}_{0}(J)=\underline{A}_{0}(J)<\infty$. Thus $\mathcal{E}_{0}(J)=\infty$.

(2) If $a_{n}=1-\frac{1}{n}, b_{n}=0$, then $Z(J)=\infty$ (by Corollary 6.3) $\bar{A}_{0}(J)=$ $\underline{A}_{0}(J)=\infty$, but $\mathcal{E}_{0}(J)<\infty$ since $J$ has no spectrum outside $[-2,2]$.

(3) If $a_{n}=1+\frac{1}{n}, b_{n}=0$, then $Z(J)<\infty$ (by Theorem 6.4), but $\bar{A}_{0}(J)=\underline{A}_{0}(J)=-\infty$ and so $\mathcal{E}_{0}(J)=\infty$.

Finally, we note that Nevai's [12] $(-1)^{n} / n$ theorem shows that we can have $Z(J)<\infty, \mathcal{E}_{0}(J)<\infty$, and have the sums $\sum a_{n}$ and/or $\sum b_{n}$ be only conditionally and not absolutely convergent.

\section{REFERENCES}

[1] R. Askey and M. Ismail, Recurrence relations, continued fractions, and orthogonal polynomials, Mem. Amer. Math. Soc. 49 (1984).

[2] K.M. Case, Orthogonal polynomials. II, J. Math. Phys. 16 (1975), 1435-1440.

[3] J. Charris and M.E.H. Ismail, On sieved orthogonal polynomials, V. Sieved Pollaczek polynomials, SIAM J. Math. Anal. 18 (1987), 1177-1218.

[4] P. Deift and R. Killip, On the absolutely continuous spectrum of one-dimensional Schrödinger operators with square summable potentials, Comm. Math. Phys. 203 (1999), 341-347.

[5] S.A. Denisov, On the coexistence of absolutely continuous and singular continuous components of the spectral measure for some Sturm-Liouville operators with square summable potentials, preprint.

[6] J. Dombrowski and P. Nevai, Orthogonal polynomials, measures and recurrence relations, SIAM J. Math. Anal. 17 (1986), 752-759.

[7] A. Figotin and L. Pastur, Spectra of random and almost-periodic operators, Springer-Verlag, Berlin, 1992.

[8] A.A. Gončar, On convergence of Padé approximants for some classes of meromorphic functions, Math. USSR Sb. 26 (1975), 555-575. 
[9] D. Hundertmark and B. Simon, Lieb-Thirring inequalities for Jacobi matrices, J. Approx. Theory, to appear.

[10] R. Killip and B. Simon, Sum rules for Jacobi matrices and their applications to spectral theory, Ann. of Math., to appear.

[11] A. Laptev, S. Naboko, and O. Safronov, in preparation.

[12] P. Nevai, Orthogonal polynomials defined by a recurrence relation, Trans. Amer. Math. Soc. 250 (1979), 369-384.

[13] P. Nevai, Orthogonal polynomials, Mem. Amer. Math. Soc. 18 (1979), no. 213, 185 pp.

[14] P. Nevai, Géza Freud, orthogonal polynomials and Christoffel functions. A case study, J. Approx. Theory 48 (1986), 3-167.

[15] E.M. Nikishin, Discrete Sturm-Liouville operators and some problems of function theory, J. Sov. Math. 35 (1986), 2679-2744.

[16] F. Peherstorfer and P. Yuditskii, Asymptotics of orthonormal polynomials in the presence of a denumerable set of mass points, Proc. Amer. Math. Soc. 129 (2001), 3213-3220.

[17] F. Pollaczek, Sur une généralisation des polynomes de Legendre, C. R. Acad. Sci. Paris 228 (1949), 1363-1365.

[18] F. Pollaczek, Systèmes de polynomes biorthogonaux qui généralisent les polynomes ultrasphériques, C. R. Acad. Sci. Paris 228 (1949), 1998-2000.

[19] F. Pollaczek, Sur une famille de polynômes orthogonaux qui contient les polynômes d'Hermite et de Laguerre comme cas limites, C. R. Acad. Sci. Paris 230 (1950), 1563-1565.

[20] W. Rudin, Real and Complex Analysis, 3rd edition, Mc-Graw Hill, New York, 1987.

[21] J.A. Shohat, Théorie Générale des Polinomes Orthogonaux de Tchebichef, Mémorial des Sciences Mathématiques, Vol. 66, pp. 1-69, Paris, 1934.

[22] B. Simon, The classical moment problem as a self-adjoint finite difference operator, Adv. Math. 137 (1998), 82-203.

[23] G. Szegö, Beiträge zue Theorie der Toeplitzschen Formen, I, II, Math. Z. 6 (1920), 167-202; 9 (1921), 167-190.

[24] G. Szegö, On certain special sets of orthogonal polynomials, Proc. Amer. Math. Soc. 1 (1950), 731-737.

[25] G. Szegő, Orthogonal Polynomials, 4th edition. American Mathematical Society, Colloquium Publications, Vol. XXIII. American Mathematical Society, Providence, R.I., 1975.

[26] S. Verblunsky, On positive harmonic functions, Proc. London Math. Soc. 40 (1935), 290-320.

[27] A. Zlatoš, in preparation.

Mathematics 253-37, California Institute of Technology, Pasadena, CA 91125

E-mail address: bsimon@caltech.edu; andrej@caltech.edu 